\title{
On nonlocal reductions of the multi-component nonlinear Schrödinger equation on symmetric spaces
}

\author{
Georgi G. Grahovski ${ }^{1}$, Junaid I. Mustafa ${ }^{2}$ and Hadi Susanto $^{3}$ \\ Department of Mathematical Sciences, University of Essex, Wivenhoe Park, Colchester, UK
}

\begin{abstract}
The aim of this paper is to develop the inverse scattering transform (IST) for multi-component generalisations of nonlocal reductions of the nonlinear Schrödinger (NLS) equation with $\mathcal{P T}$ symmetry related to symmetric spaces. This includes: the spectral properties of the associated Lax operator, Jost function, the scattering matrix and the minimal set of scattering data, the fundamental analytic solutions. As main examples we use the Manakov vector Schrödinger equation (related to A.III-symmetric spaces) and the multi-component NLS (MNLS) equations of KullishSklyanin type (related to BD.I-symmetric spaces). Furthermore, the 1- and 2-soliton solutions are obtained by using an appropriate modification of the Zakharov-Shabat dressing method. It is shown, that the MNLS equations of these types allow both regular and singular soliton configurations. Finally, we present here different examples of 1- and 2-soliton solutions for both types of models, subject to different reductions.
\end{abstract}

\section{Introduction}

One of the most important and popular completely integrable nonlinear PDE is the nonlinear Schrödinger (NLS) equation [65, 17, 63, 41]:

$$
\mathrm{i} q_{t}+q_{x x}+2|q|^{2} q(x, t)=0
$$

Here $q(x, t)$ is a complex valued function, tending fast enough to zero as $|x| \rightarrow \infty[41,6]$. It has been derived as a governing equation describing processes and phenomena in such diverse fields as deep water waves, plasma physics and nonlinear fibre optics. For instance, in optics, NLS models wave propagation in Kerr media, where the nonlinearity is proportional to the intensity of the field.

The NLS equation (1) appeared at the early stage of the development of the inverse scattering method (ISM) and the theory of solitons [6, 15, 17, 41, 63], and exhibits all remarkable properties of PDEs and systems of PDEs, integrable by the ISM: it allows soliton solutions, infinite set of integrals of motion, multi-Hamiltonian formulation and so on $[41,17]$. The key tool in studying integrability by the ISM is the existence of Lax representation of the nonlinear evolutionary equation (NLEE), that is, the NLEE can be represented as a compatibility condition of two linear operators [63, 17, 65, 41, 22]. The scattering problem for (1) is given by the Zakharov-Shabat system (related to $s l(2, \mathbb{C})$ algebra):

$$
\begin{aligned}
L \chi & \equiv\left(\mathrm{i} \frac{d}{d x}+q(x, t)-\lambda \sigma_{3}\right) \chi(x, t, \lambda)=0, \\
q(x, t) & =\left(\begin{array}{cc}
0 & q^{+} \\
q^{-} & 0
\end{array}\right), \quad \sigma_{3}=\left(\begin{array}{cc}
1 & 0 \\
0 & -1
\end{array}\right),
\end{aligned}
$$

\footnotetext{
${ }^{1}$ E-mail: graheessex.ac.uk

${ }^{2}$ E-mail: jimusteessex.ac.uk

${ }^{3}$ E-mail: hsusanto@essex.ac.uk
} 
The first integrable multi-component generalisation of the scalar NLS equation (1) is the Manakov vector NLS equation:

$$
\begin{array}{r}
\mathrm{i} q_{1, t}+q_{1, x x}+2\left(\left|q_{1}\right|^{2}+\left|q_{2}\right|^{2}\right) q_{1}(x, t)=0 \\
\mathrm{i} q_{2, t}+q_{2, x x}+2\left(\left|q_{1}\right|^{2}+\left|q_{2}\right|^{2}\right) q_{2}(x, t)=0 .
\end{array}
$$

Here, $q(x, t)$ is a 2-component vector-valued function. It is associated with a scattering problem of Zakharov-Shabat type related to the algebra $\operatorname{sl}(3, \mathbb{C})$. It was proposed by S. V. Manakov [53] as an asymptotic model for the propagation of the electric field in a waveguide. Subsequently, this system was derived as a key model for light propagation in optical fibres [54, 7].

The Manakov model (3) can be generalised to $n$-component vectors (see [63])

$$
i \mathbf{v}_{t}+\mathbf{v}_{x x}+2\left(\mathbf{v}^{\dagger}, \mathbf{v}\right) \mathbf{v}=0, \quad \mathbf{v}=\mathbf{v}(x, t) .
$$

Here $\mathbf{v}$ is an $n$-component complex-valued vector and $(\cdot, \cdot)$ is the standard scalar product. It is again integrable by the ISM $[6,63,17,41]$.

The classical ZS system can be generalised to a matrix (multi-component) form in a number of ways. One of the standard ways for doing this is by considering Lax operators taking values in a simple Lie algebra $\mathfrak{g}$ :

$$
L=\mathrm{i} \partial_{x}+Q-\lambda \mathrm{J}
$$

Here, $\mathrm{J}$ is a constant element of simple Lie algebra $\mathfrak{g}[48,42]$. Generalizations of the NLS equation to symmetric spaces related to Lie algebra $\mathfrak{g}$ are proposed in $[19,8,18]$. This includes coupled NLS systems with Lax pairs related the symmetric spaces A.III, C.I, D.III and BD.I types in the Cartan classification [48, 52]. Among this class, one can get as a special case the Manakov vector NLS equation and the one studied by Kulish and Sklyanin [50]. All these generalizations are solvable by the ISM [50, 19, 41].

When the rank $r$ of the underlying simple Lie algebra $\mathfrak{g}$ grows, the corresponding generic NLEE (or systems) will contain as many independent complex-valued functions as the number of all roots of $\mathfrak{g}[16,29,30]$. They are solvable for any $r$ but their possible applications to physics for large $r$ do not seem realistic. However one still may extract new integrable and physically useful NLEE by imposing reductions on $L(t)$, i.e. algebraic restrictions on $Q(x, t)$ which diminish the number of independent functions in them and the number of equations [57]. Of course, such restrictions must be compatible with the dynamics of the NLEE [29, 30].

Recently, in [2] was proposed a nonlocal integrable equation of nonlinear Schrödinger type

$$
\mathrm{i} q_{t}+q_{x x}+V(x, t) q(x, t)=0, \quad V(x, t)=2 q(x, t) q^{*}(-x, t) .
$$

with $\mathcal{P} \mathcal{T}$-symmetry, due to the invariance of the so-called self-induced potential $V(x, t)$ under the combined action of parity and time reversal symmetry. In the same paper, the 1-soliton solution for this model is derived and it was shown that it develops singularities in finite time. Soon after this, nonlocal $\mathcal{P} \mathcal{T}$-symmetric generalisations are found for the Ablowitz-Ladik model in [3]. All these models are integrable by the Inverse Scattering Method (ISM) [4, 40, 64, 65]. Such nonlocal reductions of the NLS equation and its multi-component generalisations are of particular interest in regards to applications in $\mathcal{P T}$-symmetric optics, especially in developing of theory of electromagnetic waves in artificial heterogenic media [1, 9, 10, 60]. For an up-to-date review, see for example [66].

Historically the first pseudo-hermitian hamiltonian with real spectrum is the $\mathcal{P} \mathcal{T}$-symmetric one in [11]. Pseudo-hermiticity here means that the Hamiltonian $\mathcal{H}$ commutes with the operators of spatial reflection $\mathcal{P}$ and time reversal $\mathcal{T}: \mathcal{P} \mathcal{T H}=\mathcal{H} \mathcal{P} \mathcal{T}[55,56]$. The action of these operators is defined as 
follows: $\mathcal{P}: x \rightarrow-x$ and $\mathcal{T}: t \rightarrow-t[12,21]$. Supposing that the wave function is a scalar, this leads to the following action of the operator of spatial reflection on the space of states: $\mathcal{P} \psi(x, t)=\psi(-x, t)$ and $\mathcal{T} \psi(x, t)=\psi^{*}(x,-t)$. As a result, the Hamiltonian and the wave function are $\mathcal{P} \mathcal{T}$-symmetric, if $\mathcal{H}(x, t)=\mathcal{H}^{*}(-x,-t)$ and $\psi(x, t)=\psi^{*}(-x,-t)$ [20]. Here we used also, that the parity operator $\mathcal{P}$ is linear and unitary while the time reversal operator $\mathcal{T}$ is anti-linear and anti-unitary.

The aim of this paper is study nonlocal reductions and to derive the corresponding soliton solutions for multi-component NLS models related to symmetric spaces of A.III-type and BD.I-type. This will be done based on examples of the vector NLS equations related to symmetric spaces of A.III $\simeq$ $S U(s+p) / S(U(s) \otimes U(p))$ type and the Kulish-Sklyanin model related to symmetric spaces of BD.I $\simeq S O(2 r+1) / S O(2) \otimes S O(2 r-1)$ type.

The structure of this paper is as follows: In Section 2 we outline the form of the Lax operators and the general form of the NLEEs as well as the nonlocal symmetries (involutions) of interest. In Section 3 we present the direct scattering problem: the Jost solutions, the scattering matrix and the minimal set of scattering data and the fundamental analytic solutions (FAS). Furthermore, In Section 4, based on appropriate modification of the Zakharov-Shabat dressing method, we derive 1- and 2soliton solution of the corresponding NLEE equation related to A.III and BD.I symmetric spaces and study the effect on nonlocal reductions on them.

\section{Preliminaries}

\subsection{Lax pair and general form of the equations}

Let us start with the generic Lax pair for the MNLS equations on symmetric spaces [23], it can be represented by the following form:

$$
\begin{aligned}
L \chi(x, t, \lambda) & \equiv \mathrm{i} \partial_{\mathrm{x}} \chi+(\mathrm{Q}(\mathrm{x}, \mathrm{t})-\lambda \mathrm{J}) \chi(\mathrm{x}, \mathrm{t}, \lambda)=0, \quad \mathrm{U}(\mathrm{x}, \mathrm{t}, \lambda)=\mathrm{Q}(\mathrm{x}, \mathrm{t})-\lambda \mathrm{J}, \\
M \chi(x, t, \lambda) & \equiv \mathrm{i} \partial_{\mathrm{t}} \chi+\left(\mathrm{V}_{0}(\mathrm{x}, \mathrm{t})+\lambda \mathrm{V}_{1}(\mathrm{x}, \mathrm{t})-\lambda^{2} \mathrm{~J}\right) \chi(\mathrm{x}, \mathrm{t}, \lambda)=0, \\
V_{1}(x, t) & =Q(x, t), \quad V_{0}(x, t)=\operatorname{iad}_{\mathrm{J}}^{-1} \frac{\mathrm{d} Q}{\mathrm{~d} x}+\frac{1}{2}\left[\operatorname{ad}_{\mathrm{J}}^{-1} Q, Q(x, t)\right] .
\end{aligned}
$$

Here $\chi(x, t, \lambda)$ are the eigenfunction of the Lax operators, $U(x, t, \lambda)$ and $V(x, t, \lambda)$ are chosen to take values in a simple Lie algebra $\mathfrak{g}$ of rank $r$, while the eigenfunctions $\chi(x, t, \lambda)$ belong to the corresponding Lie group $\mathfrak{G}$. Here also $\mathrm{J}$ is a constant element of the Cartan subalgebra $\mathfrak{h} \subset \mathfrak{g}$ (which can be always chosen to be represented by a diagonal matrix) and $\lambda \in \mathbb{C}$ is a spectral parameter. On the A.III $\simeq S U(p+s) / S(U(p) \times U(s))$ symmetric spaces the potential of $L(\lambda)$ (7a) reads:

$$
Q(x, t)=\left(\begin{array}{cc}
0 & \mathbf{q}^{+} \\
\mathbf{q}^{-} & 0
\end{array}\right), \quad \mathrm{J}=\frac{2}{s+p}\left(\begin{array}{cc}
p \mathbb{1}_{s} & 0 \\
0 & -s \mathbb{1}_{p}
\end{array}\right),
$$

where $\boldsymbol{q}^{+}(x, t)$ and $\left(\boldsymbol{q}^{-}\right)^{T}(x, t)$ are $s \times p$ matrix-valued functions belonging to the simple Lie algebra $\mathfrak{g}, \mathbb{1}_{s}$ and $\mathbb{1}_{p}$ are the $s \times s$ and $p \times p$ identity matrices, respectively, $s+p=n$.

The NLEE can be written as a compatibility condition

$$
[L(\lambda), M(\lambda)]=0,
$$

of the two Lax operators (7a)-(7b). In particular, if $L(\lambda)$ and $M(\lambda)$ are related to $\mathbf{A} . \mathbf{I I I} \simeq S U(p+$ $1) / S(U(1) \otimes U(p))$ - symmetric spaces [19], the explicit parametrisation (8) of the A.III symmetric spaces will give the system

$$
\begin{array}{r}
\mathbf{i q}_{t}^{+}+\mathbf{q}_{x x}^{+}+2 \mathbf{q}^{+} \mathbf{q}^{-} \mathbf{q}^{+}(x, t)=0 \\
-\mathbf{i} \mathbf{q}_{t}^{-}+\mathbf{q}_{x x}^{-}+2 \mathbf{q}^{-} \mathbf{q}^{+} \mathbf{q}^{-}(x, t)=0 .
\end{array}
$$


The particular choice $s=1$ and $p=2$ (assuming also the standard involution $\mathbf{q}^{-}=\left(\mathbf{q}^{+}\right)^{*}$ for the NLS type of models) corresponds to the well-know Manakov system [53]. Its generalisations for $n$-dimensional vectors $\mathbf{q}^{ \pm}$is known as vector nonlinear Schrödinger equation [41].

Another class of the multi-component NLS (MNLS) equations is known as Kulish and Sklyanin $(\mathrm{KS})$ models and are related to the symmetric spaces of BD.I $\simeq S O(2 r+1) / S O(2) \otimes S O(2 r-1)$ type $[36,25,24,28,26]$. The generic NLEEs of this class can be written as:

$$
\begin{aligned}
& \mathrm{i} \overrightarrow{\mathbf{q}}_{t}^{+}+\overrightarrow{\mathbf{q}}_{x x}^{+}+2\left(\overrightarrow{\mathbf{q}}^{+}, \overrightarrow{\mathbf{q}}^{-}\right) \overrightarrow{\mathbf{q}}^{+}-\left(\overrightarrow{\mathbf{q}}^{+}, s_{0} \overrightarrow{\mathbf{q}}^{+}\right) s_{0} \overrightarrow{\mathbf{q}}^{-}=0, \\
& \mathrm{i} \overrightarrow{\mathbf{q}}_{t}^{-}-\overrightarrow{\mathbf{q}}_{x x}^{-}-2\left(\overrightarrow{\mathbf{q}}^{+}, \overrightarrow{\mathbf{q}}^{-}\right) \overrightarrow{\mathbf{q}}^{-}+\left(\overrightarrow{\mathbf{q}}^{-}, s_{0} \overrightarrow{\mathbf{q}}^{-}\right) s_{0} \overrightarrow{\mathbf{q}}^{+}=0,
\end{aligned}
$$

which is associated with $\mathfrak{g} \simeq s o(2 r+1, \mathbb{C})$ linear system $(7 \mathrm{a})$ where

$$
Q(x, t)=\left(\begin{array}{ccc}
0 & \left(\mathbf{q}^{+}\right)^{\mathbf{T}} & 0 \\
\mathbf{q}^{-} & \mathbf{0} & s_{0} \mathbf{q}^{+} \\
0 & \left(\mathbf{q}^{-}\right)^{\mathbf{T}} s_{0} & 0
\end{array}\right), \quad \mathrm{J}=\operatorname{diag}(1, \mathbf{0},-1) .
$$

Here $\mathbf{q}^{ \pm}$are $2 r-1$-component vectors, and $s_{0}$ is the matrix that defines the orthogonal algebra $\mathbf{B}_{r} \simeq s o(2 r+1)$ :

$$
X \in s o(2 r+1) \text { iff } X+S_{0} X^{T} S_{0}^{-1}=0, \quad S_{0}=\sum_{s=1}^{2 r+1}(-1)^{s+1} E_{s, 2 r+2-s}^{(2 r+1)}=\left(\begin{array}{ccc}
0 & 0 & 1 \\
0 & -s_{0} & 0 \\
1 & 0 & 0
\end{array}\right),
$$

where $E_{s, p}^{(2 r+1)}$ is a $2 r+1 \times 2 r+1$ matrix with matrix elements given by $\left(E_{s, p}^{(2 r+1)}\right)_{i j}=\delta_{s i} \delta_{p j}$.

\subsection{Symmetries of the Lax operator and reductions}

A systematic way of description and classification of the class of admissible reductions for a given Lax pair is introduced by A. Mikhailov in [57]. This is done by the so-called reduction group. The reduction group is a finite group which preserves the Lax representation, i.e. it ensures that the reduction constraints are automatically compatible with the evolution [29, 43, 30, 62]. The reduction constrains diminish the number of independent functions and the number of equations. Here we will restrict ourselves with $\mathbb{Z}_{2}$ reductions of the following two types:

$$
\begin{array}{r}
U(x, t, \lambda)=B\left(U\left(x, t, \lambda^{*}\right)\right)^{\dagger} B^{-1}, \\
-U(x, t, \lambda)=B\left(U\left(-x, t,-\lambda^{*}\right)\right)^{\dagger} B^{-1},
\end{array}
$$

where $B$ is constant block-diagonal matrix of the form $B=\left(\begin{array}{cc}\mathbf{B}_{+} & 0 \\ 0 & \mathbf{B}_{-}\end{array}\right)$for A.III symmetric spaces. For symmetric spaces of type BD.I it can be written in the form:

$$
B=\left(\begin{array}{ccc}
\mathbf{B}_{+} & 0 & 0 \\
0 & \mathbf{B}_{ \pm} & 0 \\
0 & 0 & \mathbf{B}_{-}
\end{array}\right)
$$

Here we assume also that the blocks $\mathbf{B}_{+}$and $\mathbf{B}_{-}$are nonsingular matrices. Note also, that the involution of type (B) in (16) results in nonlocal reduction conditions.

Recalling the standard block-structure on A.III symmetric spaces (8) one can write explicitly the reductions conditions for the matrix blocks:

$$
\begin{array}{r}
\mathbf{q}^{-}(x, t)=\mathbf{B}_{-}\left(\mathbf{q}^{+}(x, t)\right)^{\dagger}\left(\mathbf{B}_{+}\right)^{-1}, \quad \mathbf{q}^{+}(x, t)=\mathbf{B}_{+}\left(\mathbf{q}^{-}(x, t)\right)^{\dagger}\left(\mathbf{B}_{-}\right)^{-1} \\
\mathbf{q}^{-}(x, t)=-\mathbf{B}_{-}\left(\mathbf{q}^{+}(-x, t)\right)^{\dagger}\left(\mathbf{B}_{+}\right)^{-1}, \quad \mathbf{q}^{+}(x, t)=-\mathbf{B}_{+}\left(\mathbf{q}^{-}(-x, t)\right)^{\dagger}\left(\mathbf{B}_{-}\right)^{-1}
\end{array}
$$


Using the the local and nonlocal involutions (18) and (19) respectively, one can write the resulting Kulish-Sklyanin models. The local involution(18) will give the following equations:

$$
\mathrm{i} \mathbf{q}_{t}^{+}+\mathbf{q}_{x x}^{+}+2\left(\mathbf{q}^{+}(x, t),\left(\mathbf{q}^{+}(x, t)\right)^{*}\right) \mathbf{q}^{+}-\left(\mathbf{q}^{+}, s_{0} \mathbf{q}^{+}\right) s_{0}\left(\mathbf{q}^{+}(x, t)\right)^{*}=0,
$$

while the nonlocal involution (19) will result in:

$$
\mathrm{i} \mathbf{q}_{t}^{+}+\mathbf{q}_{x x}^{+}+2\left(\mathbf{q}^{+}(x, t),\left(\mathbf{q}^{+}(-x, t)\right)^{*}\right) \mathbf{q}^{+}-\left(\mathbf{q}^{+}, s_{0} \mathbf{q}^{+}\right) s_{0}\left(\mathbf{q}^{+}(-x, t)\right)^{*}=0 .
$$

Example A. [Manakov model] If $\mathfrak{g} \simeq \operatorname{sl}(3, \mathbb{C}), p=1$ and $s=2$, then $\boldsymbol{q}^{+}(x, t)$ and $\boldsymbol{q}^{-}(x, t)$ are 2-component vector functions. In addition, If we apply a (local) reduction of type $(A)$ with $B=\mathbb{I}$, this will reproduce the standard Manakov VNLS equation:

$$
\begin{aligned}
& -\mathrm{i} q_{1, t}+q_{1, x x}+2\left(\left|q_{1}(x, t)\right|^{2}+\left|q_{2}(x, t)\right|^{2}\right) q_{1}(x, t)=0, \\
& -\mathrm{i} q_{2, t}+q_{2, x x}+2\left(\left|q_{1}(x, t)\right|^{2}+\left|q_{2}(x, t)\right|^{2}\right) q_{2}(x, t)=0 .
\end{aligned}
$$

Taking an involution of type $(B)$ with $\mathbf{B}_{+}=\mp 1$ and $\mathbf{B}_{-}=\operatorname{diag}( \pm 1, \pm 1)$, then we obtain the following nonlocal reduction of Manakov model:

$$
\begin{aligned}
& -\mathrm{i} q_{1, t}+q_{1, x x}+2\left(q_{1}(x, t) q_{1}^{*}(-x, t)+q_{2}(x, t) q_{2}^{*}(-x, t)\right) q_{1}(x, t)=0, \\
& -\mathrm{i} q_{2, t}+q_{2, x x}+2\left(q_{1}(x, t) q_{1}^{*}(-x, t)+q_{2}(x, t) q_{2}^{*}(-x, t)\right) q_{2}(x, t)=0 .
\end{aligned}
$$

Example B. [Kulish-Sklyanin model] In the simplest case of Lax operators related to $S O(5) / S O(2) \otimes$ $S O(3)(\operatorname{rank} \mathfrak{g}=2)$, we can set $\mathbf{q}^{+}=\left(q_{12}^{+}, q_{13}^{+}, q_{14}^{+}\right)$and $\mathbf{q}^{-}=\left(q_{12}^{-}, q_{13}^{-}, q_{14}^{-}\right)$. After assuming also the standard involution of type (A) with $B=\mathbb{I}$, the compatibility condition (9) will lead to the following 3-component NLS system:

$$
\begin{aligned}
& \mathrm{i}\left(q_{t}^{+}\right)_{12}+\left(q_{x x}^{+}\right)_{12}+2\left(\left|q_{12}\right|^{2}+2\left|q_{13}\right|^{2}\right) q_{12}+2\left(q_{14}^{+}\right)^{*}\left(q_{13}^{+}\right)^{2}=0, \\
& \mathrm{i}\left(q_{t}^{+}\right)_{13}+\left(q_{x x}^{+}\right)_{13}+2\left(\left|q_{12}\right|^{2}+\left|q_{13}\right|^{2}+\left|q_{14}\right|^{2}\right) q_{13}+2\left(q_{13}^{+}\right)^{*} q_{14}^{+} q_{12}^{+}=0, \\
& \mathrm{i}\left(q_{t}^{+}\right)_{14}+\left(q_{x x}^{+}\right)_{14}+2\left(\left|q_{14}\right|^{2}+2\left|q_{13}\right|^{2}\right) q_{14}+2\left(q_{12}^{+}\right)^{*}\left(q_{13}^{+}\right)^{2}=0 .
\end{aligned}
$$

This appears to be a model describing $\mathcal{F}=1$ spinor Bose-Einstein condensates in one-dimensional approximation [50, 26, 37, 45].

\section{Direct Scattering Transform for $L(\lambda)$}

\subsection{Jost solutions and scattering matrix}

The starting point here are the so-called Jost solutions, which are determined by their asymptotics for $|x| \rightarrow \infty$ :

$$
\lim _{x \rightarrow \infty} \mathrm{e}^{\mathrm{i} \lambda \mathrm{J} x} \psi(x, t, \lambda)=\mathbb{1}_{s+p}, \quad \lim _{x \rightarrow-\infty} \mathrm{e}^{\mathrm{i} \lambda \mathrm{J} x} \phi(x, t, \lambda)=\mathbb{1}_{s+p}, \quad \lambda \in \mathbb{R} .
$$

Along with these functions, one can also use "normalised to unity" Jost solutions

$$
\xi(x, t, \lambda)=\psi(x, t, \lambda) \mathrm{e}^{(\mathrm{i} \lambda J x)}, \quad \varphi(x, t, \lambda)=\phi(x, t, \lambda) \mathrm{e}^{(\mathrm{i} \lambda \mathrm{J} x)},
$$

satisfying the following equation

$$
\mathrm{i} \frac{\mathrm{d} \xi}{\mathrm{d} x}+Q(x, t) \xi(x, t, \lambda)-\lambda[\mathrm{J}, \xi(x, t, \lambda)]=0,
$$


provided that (25) satisfy (7a).

On the continuous spectrum of $L(\lambda)$ the two Jost solutions $\psi(x, t, \lambda)$ and $\phi(x, t, \lambda)$ are related via scattering matrix $T(\lambda)$

$$
\phi(x, t, \lambda)=\psi(x, t, \lambda) T(\lambda), \quad \lambda \in \mathbb{R} .
$$

The scattering matrix $T(\lambda)$ belongs to the Lie group $\mathfrak{G}$ corresponding the Lie algebra /symmetric space of $L(\lambda)$. For symmetric spaces of type A.III in the Cartan classification, it has the following block structure:

$$
T(\lambda)=\left(\begin{array}{cc}
\boldsymbol{a}^{+}(\lambda) & -\boldsymbol{b}^{-}(\lambda) \\
\boldsymbol{b}^{+}(\lambda) & \boldsymbol{a}^{-}(\lambda)
\end{array}\right)
$$

where, $\boldsymbol{a}^{+}(\lambda)$ and $\boldsymbol{a}^{-}(\lambda)$ are square matrices, while $\boldsymbol{b}^{+}(\lambda)$ and $\boldsymbol{b}^{-}(\lambda)$ are rectangular matrices. The block structure of the inverse of $T(\lambda)$ can be written as:

$$
\hat{T}(\lambda)=\left(\begin{array}{cc}
\boldsymbol{c}^{-}(\lambda) & \boldsymbol{d}^{-}(\lambda) \\
-\boldsymbol{d}^{+}(\lambda) & \boldsymbol{c}^{+}(\lambda)
\end{array}\right), \quad \hat{T}(\lambda)=T^{-1}(\lambda)
$$

where

$$
\begin{aligned}
& \boldsymbol{c}^{ \pm}(\lambda)=\hat{\boldsymbol{a}}^{\mp}(\lambda)\left(\mathbb{1}+\rho^{ \pm} \rho^{\mp}\right)^{-1}=\left(\mathbb{1}+\tau^{\mp} \tau^{ \pm}\right)^{-1} \hat{\boldsymbol{a}}^{\mp}(\lambda), \\
& \boldsymbol{d}^{ \pm}(\lambda)=\hat{\boldsymbol{a}}^{\mp}(\lambda) \rho^{ \pm}(\lambda)\left(\mathbb{1}+\rho^{\mp} \rho^{ \pm}\right)^{-1}=\left(\mathbb{1}+\tau^{\mp} \tau^{ \pm}\right)^{-1} \tau^{\mp}(\lambda) \hat{\boldsymbol{a}}^{ \pm}(\lambda) .
\end{aligned}
$$

Here $\rho^{ \pm}(\lambda)$ and $\tau^{ \pm}(\lambda)$ are the reflection and transmission coefficients respectively:

$$
\rho^{ \pm}(\lambda)=\mathbf{b}^{ \pm} \hat{\mathbf{a}}^{ \pm}(\lambda)=\hat{\mathbf{c}}^{ \pm} \mathbf{d}^{ \pm}(\lambda), \quad \tau^{ \pm}(\lambda)=\hat{\mathbf{a}}^{ \pm} \mathbf{b}^{\mp}(\lambda)=\mathbf{d}^{\mp} \hat{\mathbf{c}}^{ \pm}(\lambda) .
$$

For symmetric spaces of BD.I type, the block structure of $T(t, \lambda)$ and its inverse take the form:

$$
T(t, \lambda)=\left(\begin{array}{ccc}
m_{1}^{+} & -\overrightarrow{\mathbf{B}}^{-T} & c_{(1)}^{-} \\
\overrightarrow{\mathbf{b}}^{+} & \mathbf{T}_{22} & -s_{0} \overrightarrow{\mathbf{b}}^{-} \\
c_{(1)}^{+} & \overrightarrow{\mathbf{B}^{+T}} s_{0} & m_{1}^{-}
\end{array}\right), \quad \hat{T}(t, \lambda)=\left(\begin{array}{ccc}
m_{1}^{-} & \overrightarrow{\mathbf{b}}^{-T} & c_{(1)}^{-} \\
-\overrightarrow{\mathbf{B}}^{+} & \hat{\mathbf{T}}_{22} & s_{0} \overrightarrow{\mathbf{B}}^{-} \\
c_{(1)}^{+} & -\overrightarrow{\mathbf{b}}^{+T} s_{0} & m_{1}^{+}
\end{array}\right) .
$$

Here, $\overrightarrow{\mathbf{B}}^{ \pm}(t, \lambda)$ and $\overrightarrow{\mathbf{b}}^{ \pm}(t, \lambda)$ are $2 r-1$-component vectors and $c_{(1)}^{ \pm}(\lambda)$ and $m_{1}^{ \pm}(\lambda)$ are scalar functions. The reflection coefficients $\vec{\rho}^{ \pm}(\lambda)$, the transmission coefficients $\vec{\tau}^{ \pm}(\lambda)$ and the functions $c_{(1)}^{ \pm}(\lambda)$ are determined by the generalised Gauss decomposition of $T(t, \lambda)$ :

$$
T(t, \lambda)=T_{\mathrm{J}}^{-}(t, \lambda) D_{\mathrm{J}}^{+}(t, \lambda) \hat{S}_{\mathrm{J}}^{+}(t, \lambda)=T_{\mathrm{J}}^{+}(t, \lambda) D_{\mathrm{J}}^{-}(t, \lambda) \hat{S}_{\mathrm{J}}^{-}(t, \lambda) .
$$

Here, $S_{\mathrm{J}}^{ \pm}$and $T_{\mathrm{J}}^{ \pm}$are upper- and lower- block-triangular matrices, which can be written in the form:

$$
S_{\mathrm{J}}^{ \pm}=\mathrm{e}^{\left( \pm\left(\vec{\tau}^{ \pm}(\lambda, t) \cdot \vec{E}_{1}^{ \pm}\right)\right)}, \quad T_{\mathrm{J}}^{ \pm}=\mathrm{e}^{\left(\mp\left(\vec{\rho}^{\mp}(\lambda, t) \cdot \vec{E}_{1}^{ \pm}\right)\right)},
$$

where

$$
\vec{\tau}^{ \pm}(\lambda, t)=\overrightarrow{\mathbf{B}}^{\mp} / m_{1}^{ \pm}, \quad \vec{\rho}^{ \pm}(\lambda, t)=\overrightarrow{\mathbf{b}}^{ \pm} / m_{1}^{ \pm}, \quad c_{(1)}^{ \pm}=\frac{m_{1}^{ \pm}}{2}\left(\vec{\rho}^{ \pm, T}, s_{0} \vec{\rho}^{ \pm}\right)=\frac{m_{1}^{\mp}}{2}\left(\vec{\tau}^{\mp, T}, s_{0} \vec{\tau}^{\mp}\right),
$$

and $D_{\mathrm{J}}^{ \pm}(t, \lambda)$ is the block-diagonal factor in (34):

$$
D_{\mathrm{J}}^{+}=\left(\begin{array}{ccc}
m_{1}^{+} & 0 & 0 \\
0 & \mathbf{m}_{2}^{+} & 0 \\
0 & 0 & 1 / m_{1}^{+}
\end{array}\right), \quad D_{\mathrm{J}}^{-}=\left(\begin{array}{ccc}
1 / m_{1}^{-} & 0 & 0 \\
0 & \mathbf{m}_{2}^{-} & 0 \\
0 & 0 & m_{1}^{-}
\end{array}\right) .
$$


Here, $m_{k}^{ \pm}(t, \lambda)$ are the upper/lower rank $k$ principal minors of the scattering matrix $T(t, \lambda)(34)$ and

$$
\mathbf{m}_{2}^{+}=\mathbf{T}_{22}+\frac{\vec{b}^{+} \vec{B}^{-, T}}{m_{1}^{+}}, \quad \mathbf{m}_{2}^{-}=\mathbf{T}_{22}+\frac{s_{0} \vec{B}^{-} \vec{b}^{+, T} s_{0}}{m_{1}^{-}} .
$$

If the potential matrix $Q(x, t)$ satisfies the NLEE (9) then the associated scattering matrix $T(t, \lambda)$ evolves in time linearly, i.e. it satisfies the equation

$$
\mathrm{i} \frac{\mathrm{d} T}{\mathrm{~d} t}+[\mathrm{f}(\lambda), T(t, \lambda)]=0 .
$$

Here $f(\lambda)$ is the dispersion law of the NLEE (9). For the NLS type of equations, we have $f(\lambda)=$ $-\lambda^{2} J$.

\subsection{Fundamental Analytic Solutions (FAS)}

In the this section, we will briefly outline the construction of the Fundamental Analytic Solutions (FAS) $\chi(x,, t, \lambda)$ of the generalised Zakharov-Shabat system (7) [23, 41]. The FAS can be directly obtained from Jost solutions of (7):

$$
\chi^{ \pm}(x, t, \lambda)=\phi(x, t, \lambda) S_{\mathrm{J}}^{ \pm}(t, \lambda)=\psi(x, t, \lambda) T_{\mathrm{J}}^{\mp}(t, \lambda) D_{\mathrm{J}}^{ \pm}(t, \lambda)
$$

by using the generalised Gauss decomposition (34) of the scattering matrix $T(t, \lambda)$.

On the real axis (i.e. on the continuous spectrum of $L(\lambda)$ ), the two FAS are linearly dependent:

$$
\chi^{+}(x, t, \lambda)=\chi^{-}(x, t, \lambda) G_{0}(t, \lambda), \quad \lambda \in \mathbb{R},
$$

where the sewing function $G_{0}(t, \lambda)$ can be expressed in terms of the generalised Gauss factors $S_{\mathrm{J}}^{ \pm}(t, \lambda)$ :

$$
G_{0, J}(t, \lambda)=\left.\hat{S}_{J}^{-}(t, \lambda) S_{J}^{+}(t, \lambda)\right|_{t=0} .
$$

The independent matrix elements of $G_{0}(t, \lambda)$, together with the discrete spectrum of $L(\lambda)$ form up the minimal set of scattering data of $L$. Based on the completeness relations of the associated square solution and Wronskian type of relation, one can recover the potential $Q(x, t)$ out of the minimal set of scattering data [39].

We end up this section with the remark that, although the general form of the Gauss decomposition (34) holds true for any symmetric space, for symmetric spaces of type A.III one can simplify the form of the matrix block by slightly modifying the Gauss decomposition of $T(\lambda)^{4}$ :

$$
T(\lambda)=\mathbf{T}_{J}^{-}(\lambda) \hat{\mathbf{S}}_{J}^{+}(\lambda)=\mathbf{T}_{J}^{+}(\lambda) \hat{\mathbf{S}}_{J}^{-}(\lambda) .
$$

Now, $\mathbf{S}^{ \pm}(\lambda)$ and $\mathbf{T}^{ \pm}(\lambda)$ are the block-triangular matrices:

$$
\begin{array}{cc}
\mathbf{S}_{J}^{+}(\lambda)=\left(\begin{array}{cc}
\mathbb{1}_{s}, \boldsymbol{d}^{-}(\lambda) \\
0, & \boldsymbol{c}^{+}(\lambda)
\end{array}\right), & \mathbf{T}_{J}^{-}(\lambda)=\left(\begin{array}{cc}
\boldsymbol{a}^{+}(\lambda), & 0 \\
\boldsymbol{b}^{+}(\lambda), & \mathbb{1}_{p}
\end{array}\right), \\
\mathbf{S}_{J}^{-}(\lambda)=\left(\begin{array}{cc}
\boldsymbol{c}^{-}(\lambda), & 0 \\
-\boldsymbol{d}^{+}(\lambda), & \mathbb{1}_{p}
\end{array}\right), & \mathbf{T}_{J}^{+}(\lambda)=\left(\begin{array}{cc}
\mathbb{1}_{s},-\boldsymbol{b}^{-}(\lambda) \\
0, & \boldsymbol{a}^{-}(\lambda)
\end{array}\right) .
\end{array}
$$

\footnotetext{
${ }^{4}$ Decomposition of type (43) are known as LU decomposition of $T(\lambda)$, while a decomposition of type (34) is known as LDU decomposition of $T(\lambda)$.
} 
Using the Gauss decomposition (43) one can write explicitly the sewing function $G_{0, J}(t, \lambda)(42)$ in terms of the blocks of $T(t, \lambda)$ and it inverse:

$$
G_{0}(\lambda)=\hat{D}^{-}(\lambda)\left(\mathbb{1}+K^{-}(\lambda)\right), \quad \hat{G}_{0}(\lambda)=\hat{D}^{+}(\lambda)\left(\mathbb{1}-K^{+}(\lambda)\right) .
$$

Here the block-diagonal factors $D^{ \pm}(\lambda)$ and block-off-diagonal $K^{ \pm}(\lambda)$ factors are expressed in terms of the blocks $\mathbf{a}^{ \pm}(\lambda), \mathbf{b}^{ \pm}(\lambda)$ of $T(\lambda)$ (29) and in terms of the blocks $\mathbf{c}^{ \pm}(\lambda), \mathbf{d}^{ \pm}(\lambda)$ (30) of its inverse $\hat{T}(\lambda)$, respectively;

$$
\begin{array}{rlrl}
D^{-}(\lambda) & =\left(\begin{array}{cc}
\mathbf{c}^{-}(\lambda) & 0 \\
0 & \mathbf{a}^{-}(\lambda)
\end{array}\right), & K^{-}(\lambda) & =\left(\begin{array}{cc}
0 & \mathbf{d}^{-}(\lambda) \\
\mathbf{b}^{+}(\lambda) & 0
\end{array}\right), \\
D^{+}(\lambda) & =\left(\begin{array}{cc}
\mathbf{a}^{+}(\lambda) & 0 \\
0 & \mathbf{c}^{+}(\lambda)
\end{array}\right), & K^{+}(\lambda)=\left(\begin{array}{cc}
0 & \mathbf{b}^{-}(\lambda) \\
\mathbf{d}^{+}(\lambda) & 0
\end{array}\right) .
\end{array}
$$

The superscripts " $\pm^{\prime \prime}$ in the expressions $D^{ \pm}(\lambda)$ above mean analyticity for $\lambda \in \mathbb{C}_{ \pm}$.

\section{Dressing method and soliton solutions}

\subsection{The Zakharov-Shabat Dressing Method}

The main idea of the Zakharov-Shabat dressing method is to start with a regular solution $\eta_{0}^{ \pm}(x, t, \lambda)$ of (7a) and to perform a "dressing" by adding singularities at two prescribed points $\lambda_{k}^{ \pm}[49,36,31$, 27, 23, 63, 61, 13]. The new singular solution will have the form:

$$
\eta^{ \pm}(x, t, \lambda)=u_{k}(x, t, \lambda) \eta_{0}^{ \pm}(x, t, \lambda) w_{k, \pm}^{-1}(\lambda) .
$$

Here, the matrices $w_{k, \pm}(\lambda)$ can be given by:

$$
w_{k,+}(\lambda)=\mathbb{1}, \quad w_{k,-}(\lambda)=\left(\begin{array}{cc}
u_{11, k}^{+} & 0 \\
0 & u_{22, k}^{-}
\end{array}\right),
$$

where the limits of the dressing factor can be obtained by:

$$
\begin{array}{rlrl}
\lim _{x \rightarrow \infty} u_{k}(x, t, \lambda) & =\left(\begin{array}{cc}
u_{11, k}^{+} & 0 \\
0 & \mathbb{1}
\end{array}\right), & & u_{11, k}^{+}=\mathbb{1}+\left(c_{k}(\lambda)-1\right) P_{11, k}^{+}, \\
\lim _{x \rightarrow-\infty} u_{k}(x, t, \lambda) & =\left(\begin{array}{cc}
\mathbb{1} & 0 \\
0 & u_{22, k}^{-}
\end{array}\right), & u_{22, k}^{-}=\mathbb{1}+\left(c_{k}(\lambda)-1\right) P_{22, k}^{-} .
\end{array}
$$

For Lax operators relatred to A.III symmetric spaces, the dressing factors have the form:

$$
u_{k}(x, t, \lambda)=\mathbb{1}+\left(c_{k}(\lambda)-1\right) P_{k}(x, t), \quad c_{k}(\lambda)=\frac{\lambda-\lambda_{k}^{+}}{\lambda-\lambda_{k}^{-}} .
$$

Here $u_{k}(x, t, \lambda)$ is the dressing factor, $P_{k}(x, t)$ is a projector of rank 1 has the form:

$$
P_{k}(x, t)=\frac{\left|n_{k}(x, t)\right\rangle\left\langle m_{k}(x, t)\right|}{\left\langle m_{k}(x, t) \mid n_{k}(x, t)\right\rangle},
$$

where $\left|n_{k}(x, t)\right\rangle=\chi_{0}^{+}\left(x, t, \lambda_{1}^{+}\right)\left|n_{0,1}\right\rangle$ is a column vector and $\left\langle m_{1}(x, t)\right|=\left\langle m_{0,1}\right| \hat{\chi}_{0}^{-}\left(x, t, \lambda_{1}^{-}\right)$is a row vector and both of them are eigenvector. The projectors $P_{k}(x, t)$ automatically satisfy the condition $P_{k}^{2}(x, t)=P_{k}(x, t)$. The functions $\eta^{ \pm}(x, t, \lambda)$ satisfies the linear system:

$$
\left(\mathrm{i} \frac{\mathrm{d} \eta^{ \pm}}{\mathrm{d} x}+Q(x, t) \eta^{ \pm}(x, t, \lambda)-\lambda\left[\mathrm{J}, \eta^{ \pm}(x, t, \lambda)\right]\right)=0 .
$$


where $\eta^{ \pm}(x, t, \lambda)$ are related to the ZS system with an unknown potential $Q(x, t)$, which can be found later, while $\eta_{0}^{ \pm}(x, t, \lambda)$ is related to the ZS system with a known potential $Q_{0}(x, t)$ :

$$
\mathrm{i} \frac{\mathrm{d} \eta_{0}^{ \pm}}{\mathrm{d} x}+Q_{0}(x, t) \eta_{0}^{ \pm}(x, t, \lambda)-(\lambda)\left[\mathrm{J}, \eta_{0}^{ \pm}(x, t, \lambda)\right]=0 .
$$

Now, from (48a) and (55), we find that the dressing factor $u_{k}(x, t, \lambda)$ satisfies the following equation:

$$
\mathrm{i} \frac{\mathrm{d} u_{k}}{\mathrm{~d} x}+Q(x, t) u_{k}(x, t, \lambda)-u_{k}(x, t, \lambda) Q_{0}(x, t)-\lambda\left[\mathrm{J}, u_{k}(x, t, \lambda)\right]=0,
$$

since the anzats for the dressing factor $u_{k}(x, t, \lambda)$ in (52) and (56) are compatible with respect to $\lambda$, then there are two conditions can be applied to the left-hand side of (56) which are the limit for $\lambda \rightarrow \infty$ and the residue at $\lambda=\lambda_{k}^{-}$and both of them are vanish. The first condition leads to the potential:

$$
Q(x, t)-Q_{0}(x, t)=-\left(\lambda_{k}^{+}-\lambda_{k}^{-}\right)\left[\mathrm{J}, P_{k}(x, t)\right]
$$

and the second condition gives the following nonlinear equation for $P_{k}(x, t)$ :

$$
\begin{aligned}
\mathrm{i} \frac{\mathrm{d} P_{k}}{\mathrm{~d} x} & +Q_{0}(x, t) P_{k}(x, t)-P_{k}(x, t) Q_{0}(x, t)-\lambda_{k}^{+} \mathrm{J} P_{k}(x, t)+\lambda_{k}^{-} P_{k}(x, t) \mathrm{J} \\
& +\left(\lambda_{k}^{+}-\lambda_{k}^{-}\right) P_{k}(x, t) \mathrm{J} P_{k}(x)=0 .
\end{aligned}
$$

In addition, we have the normalization condition $\lim _{\lambda \rightarrow \infty} u_{k}(x, t, \lambda)=\mathbb{1}$.

In the case of BD.I symmetric spaces the dressing factor $u_{k}(x, t, \lambda)$ can be written in the form:

$$
u_{k}(x, t, \lambda)=\mathbb{1}+\left(c_{k}(\lambda)-1\right) P_{k}(x, t)+\left(\frac{1}{c_{k}(\lambda)}-1\right) \bar{P}_{k}(x, t), \quad \bar{P}_{k}=S_{0} P_{k}^{T} S_{0}^{-1},
$$

where $P_{k}(x, t)$ and $\bar{P}_{k}(x, t)$ are mutually orthogonal projectors with rank 1 [30]. In a similar fashion, one can write the dressed potential $Q(x, t)$ as:

$$
Q(x, t)=Q_{0}(x, t)-\left(\lambda_{k}^{+}-\lambda_{k}^{-}\right)\left[\mathrm{J}, P_{k}(x, t)-\bar{P}_{k}(x, t)\right] .
$$

\subsection{One Soliton Solutions}

Example 1. If we take $\mathfrak{g} \simeq s l(3, \mathbb{C})$ and a dressing factor in the form (52) satisfying the nonlocal reduction conditions (16), this will imply the following involution on the dressing factor:

$$
B u_{1}\left(-x, t,-\lambda^{*}\right)^{\dagger} B^{-1}=u_{1}^{-1}(x, t, \lambda)
$$

where $B$ is constant block-diagonal matrix. As a result, the projector $P_{1}$ must satisfy:

$$
P_{1}(x, t)=B P_{1}^{\dagger}(-x, t) B^{-1}, \quad\left(-\lambda^{ \pm}\right)^{*}=\lambda^{\mp} .
$$

That means the projector $P_{1}(x, t)$ and $c_{1}(\lambda)$ become:

$$
\begin{aligned}
P_{1}(x, t) & =\frac{\left|n_{1}(x, t)\right\rangle\left\langle n_{1}^{*}(-x, t)\right| B}{\left\langle n_{1}^{*}(-x, t)|B| n_{1}(x, t)\right\rangle}, \quad\left\langle m_{1}(x, t)\right|=\left(B\left|n_{1}(-x, t)\right\rangle\right)^{\dagger} \\
c_{1}(\lambda) & =\frac{\lambda-\lambda_{1}^{+}}{\lambda+\left(\lambda_{1}^{+}\right)^{*}}
\end{aligned}
$$


So, from (57), the components of the one-soliton solution can be written as:

$$
\begin{array}{lc}
q_{1 j}(x, t)=-2\left(\lambda_{1}^{+}+\left(\lambda_{1}^{+}\right)^{*}\right) \frac{n_{0,1}^{1}\left(n_{0,1}^{j}\right)^{*} \mathrm{e}^{-\mathrm{i}\left(\tilde{M}_{1}^{+}(x, t)+\left(\tilde{M}_{1}^{+}\right)^{*}(-x, t)\right)}}{2 R_{0,1} \cosh \left(2 \nu_{1} \mathrm{x}+2 \tilde{\mu}_{1} \mathrm{t}+\xi_{0,1}\right)}, \quad j=2,3, \\
q_{j 1}(x, t)=-2\left(\lambda_{1}^{+}+\left(\lambda_{1}^{+}\right)^{*}\right) \frac{n_{0,1}^{j}\left(n_{0,1}^{1}\right)^{*} \mathrm{e}^{\mathrm{i}\left(\tilde{M}_{1}^{+}(x, t)+\left(\tilde{M}_{1}^{+}\right)^{*}(-x, t)\right)}}{2 R_{0,1} \cosh \left(2 \nu_{1} \mathrm{x}+2 \tilde{\mu_{1}} \mathrm{t}+\xi_{0,1}\right)}, \quad j=2,3 .
\end{array}
$$

Here,

$$
\begin{aligned}
\tilde{M}_{1}^{ \pm}(x, t) & =\lambda_{1}^{ \pm} x+2 \mathrm{f}_{0,1}^{ \pm} \mathrm{t}, \quad\left(\tilde{\mathrm{M}}_{1}^{+}(-\mathrm{x}, \mathrm{t})\right)^{*}=\left(-\lambda_{1}^{+}\right)^{*}(-\mathrm{x})+2\left(\mathrm{f}_{0}\left(-\lambda_{1}^{+}\right)^{*}\right) \mathrm{t}, \\
\nu_{1} & =\mathrm{i}\left(\left(-\lambda_{1}^{+}\right)^{*}-\lambda_{1}^{+}\right) / 2, \quad \tilde{\mu}_{1}=\mathrm{i}\left(\mathrm{f}\left(-\lambda_{1}^{+}\right)^{*}-\mathrm{f}\left(\lambda_{1}^{+}\right)\right), \\
R_{0,1} & =\sqrt{\left(n_{0,1}^{1}\right)^{*} n_{0,1}^{1}\left(\left(n_{0,1}^{2}\right)^{*} n_{0,1}^{2}+\left(n_{0,1}^{3}\right)^{*} n_{0,1}^{3}\right)}, \quad \xi_{0,1}=\frac{1}{2} \ln \frac{\left(\mathrm{n}_{0,1}^{1}\right)^{*} \mathrm{n}_{0,1}^{1}}{\left(\mathrm{n}_{0,1}^{2}\right)^{*} \mathrm{n}_{0,1}^{2}+\left(\mathrm{n}_{0,1}^{3}\right)^{*} \mathrm{n}_{0,1}^{3}} .
\end{aligned}
$$

Example 2. If we take again $\mathfrak{g} \simeq s l(3, \mathbb{C})$ and the involution automorphism to be the Weyl reflection with respect to the second simple root $\mathrm{e}_{2}-\mathrm{e}_{3}$ of $\operatorname{sl}(3, \mathbb{C})$ and impose a reduction of type $(B)$ in (16). This will correspond to another block matrix $B=\left(\begin{array}{cc}\mathbf{B}_{+} & 0 \\ 0 & \mathbf{B}_{-}\end{array}\right)$where $\mathbf{B}_{-}$is a block off-diagonal matrix. The blocks can be written as

$$
\mathbf{B}_{+}=\mp 1, \quad \text { and } \quad \mathbf{B}_{-}=\left(\begin{array}{cc}
0 & \pm 1 \\
\pm 1 & 0
\end{array}\right)
$$

The reductions conditions for the matrix blocks can be written as:

$$
\mathbf{q}^{-}(x, t)=-\mathbf{B}_{-}\left(\mathbf{q}^{+}(-x, t)\right)^{\dagger}\left(\mathbf{B}_{+}\right)^{-1}, \quad \mathbf{q}^{+}(x, t)=-\mathbf{B}_{+}\left(\mathbf{q}^{-}(-x, t)\right)^{\dagger}\left(\mathbf{B}_{-}\right)^{-1} .
$$

Now, if we apply the involution of type $(C)$ with using (67), then the standard Manakov VNLS equation reproduced as:

$$
\begin{aligned}
& -\mathrm{i} q_{1, t}+q_{1, x x}+2\left(q_{1}(x, t) q_{2}^{*}(-x, t)+q_{2}(x, t) q_{1}^{*}(-x, t)\right) q_{1}(x, t)=0, \\
& -\mathrm{i} q_{2, t}+q_{2, x x}+2\left(q_{1}(x, t) q_{2}^{*}(-x, t)+q_{2}(x, t) q_{1}^{*}(-x, t)\right) q_{2}(x, t)=0 .
\end{aligned}
$$

Also, we can write one-soliton solution of the Manakov VNLS equation for this type of involution as the dressing factor (52) is satisfied by the involution (62) with (63). So, from (57), the components of the one-soliton solution can be written as:

$$
\begin{aligned}
& q_{12}(x, t)=-2\left(\lambda_{1}^{+}+\left(\lambda_{1}^{+}\right)^{*}\right) n_{0,1}^{1} \mathrm{e}^{-\mathrm{i} \tilde{M}_{1}^{+}(x, t)} R_{1}^{-1}(x, t)\left(n_{0,1}^{3}\right)^{*} \mathrm{e}^{-\mathrm{i} \tilde{M}_{1}^{+, *}(-x, t)}, \\
& q_{13}(x, t)=-2\left(\lambda_{1}^{+}+\left(\lambda_{1}^{+}\right)^{*}\right) n_{0,1}^{1} \mathrm{e}^{-\mathrm{i} \tilde{M}_{1}^{+}(x, t)} R_{1}^{-1}(x, t)\left(n_{0,1}^{2}\right)^{*} \mathrm{e}^{-\mathrm{i} \tilde{M}_{1}^{+, *}(-x, t)}, \\
& q_{21}(x, t)=-2\left(\lambda_{1}^{+}+\left(\lambda_{1}^{+}\right)^{*}\right) n_{0,1}^{2} \mathrm{e}^{\mathrm{i} \tilde{M}_{1}^{+}(x, t)} R_{1}^{-1}(x, t)\left(n_{0,1}^{1}\right)^{*} \mathrm{e}^{\left.\mathrm{i} \tilde{M}_{1}^{+, *}(-x, t)\right)}, \\
& q_{31}(x, t)=-2\left(\lambda_{1}^{+}+\left(\lambda_{1}^{+}\right)^{*}\right) n_{0,1}^{3} \mathrm{e}^{\mathrm{i} \tilde{M}_{1}^{+}(x, t)} R_{1}^{-1}(x, t)\left(n_{0,1}^{1}\right)^{*} \mathrm{e}^{\left.\mathrm{i} \tilde{M}_{1}^{+, *}(-x, t)\right)}
\end{aligned}
$$

Here,

$$
\begin{aligned}
\tilde{M}_{1}^{ \pm}(x, t) & =\lambda_{1}^{ \pm} x+2 \mathrm{f}_{0,1}^{ \pm} \mathrm{t}, \quad\left(\tilde{\mathrm{M}}_{1}^{+}(-\mathrm{x}, \mathrm{t})\right)^{*}=\left(-\lambda_{1}^{+}\right)^{*}(-\mathrm{x})+2\left(\mathrm{f}_{0}\left(-\lambda_{1}^{+}\right)^{*}\right) \mathrm{t}, \\
R_{1}(x, t) & =2 R_{0,1} \cosh \left(2 \nu_{1} \mathrm{x}+2 \tilde{\mu_{1}} \mathrm{t}+\xi_{0,1}\right), \\
\nu_{1} & =\mathrm{i}\left(\left(-\lambda_{1}^{+}\right)^{*}-\lambda_{1}^{+}\right) / 2, \quad \tilde{\mu_{1}}=\mathrm{i}\left(\mathrm{f}\left(-\lambda_{1}^{+}\right)^{*}-\mathrm{f}\left(\lambda_{1}^{+}\right)\right), \\
R_{0,1} & =\sqrt{\left(n_{0,1}^{1}\right)^{*} n_{0,1}^{1}\left(\left(n_{0,1}^{3}\right)^{*} n_{0,1}^{2}+\left(n_{0,1}^{2}\right)^{*} n_{0,1}^{3}\right)}, \quad \xi_{0,1}=\frac{1}{2} \ln \frac{\left(\mathrm{n}_{0,1}^{1}\right)^{*} \mathrm{n}_{0,1}^{1}}{\left(\mathrm{n}_{0,1}^{3}\right)^{*} \mathrm{n}_{0,1}^{2}+\left(\mathrm{n}_{0,1}^{2}\right)^{*} \mathrm{n}_{0,1}^{3}} .
\end{aligned}
$$


Example 3. If we take $\mathfrak{g} \simeq s o(5, \mathbb{C})$, then the dressing factor will take the form $(60)$. If we impose the nonlocal reduction conditions (16):

$$
B u_{1}\left(-x, t,-\lambda^{*}\right)^{\dagger} B^{-1}=u_{1}^{-1}(x, t, \lambda),
$$

this will lead to the following reduction condition:

$$
P_{1}(x, t)=B P_{1}^{\dagger}(-x, t) B^{-1}, \quad\left(-\lambda^{ \pm}\right)^{*}=\lambda^{\mp} .
$$

That means the projector $P_{1}(x, t)$ and $c_{1}(\lambda)$ become:

$$
\begin{aligned}
P_{1}(x, t) & =\frac{\left|n_{1}(x, t)\right\rangle\left\langle n_{1}^{*}(-x, t)\right| B}{\left\langle n_{1}^{*}(-x, t)|B| n_{1}(x, t)\right\rangle}, \quad\left\langle m_{1}(x, t)\right|=\left(B\left|n_{1}(-x, t)\right\rangle\right)^{\dagger}, \\
c_{1}(\lambda) & =\frac{\lambda-\lambda_{1}^{+}}{\lambda+\left(\lambda_{1}^{+}\right)^{*}} .
\end{aligned}
$$

Thus, taking the trivial solution $Q_{0}(x, t)=0$, the components of one soliton solution can be written as:

$$
\begin{aligned}
& q_{12}^{+}(x, t)=q_{45}^{+}(x, t)=-\left(\lambda_{1}^{+}+\left(\lambda_{1}^{+}\right)^{*}\right) R_{1}^{-1}(x, t)\left(n_{0,1}^{1}\left(n_{0,1}^{2}\right)^{*} \mathrm{e}^{-\mathrm{i} M_{1}^{+}(x, t)}-n_{0,1}^{4}\left(n_{0,1}^{5}\right)^{*} \mathrm{e}^{-\mathrm{i}\left(M_{1}^{+}(-x, t)\right)^{*}}\right), \\
& q_{13}^{+}(x, t)=-q_{35}^{+}(x, t)=-\left(\lambda_{1}^{+}+\left(\lambda_{1}^{+}\right)^{*}\right) R_{1}^{-1}(x, t)\left(n_{0,1}^{1}\left(n_{0,1}^{3}\right)^{*} \mathrm{e}^{-\mathrm{i} M_{1}^{+}(x, t)}+n_{0,1}^{3}\left(n_{0,1}^{5}\right)^{*} \mathrm{e}^{-\mathrm{i}\left(M_{1}^{+}(-x, t)\right)^{*}}\right), \\
& q_{14}^{+}(x, t)=q_{25}^{+}(x, t)=-\left(\lambda_{1}^{+}+\left(\lambda_{1}^{+}\right)^{*}\right) R_{1}^{-1}(x, t)\left(n_{0,1}^{1}\left(n_{0,1}^{4}\right)^{*} \mathrm{e}^{-\mathrm{i} M_{1}^{+}(x, t)}-n_{0,1}^{2}\left(n_{0,1}^{5}\right)^{*} \mathrm{e}^{-\mathrm{i}\left(M_{1}^{+}(-x, t)\right)^{*}}\right), \\
& q_{21}^{-}(x, t)=q_{54}^{-}(x, t)=-\left(\lambda_{1}^{+}+\left(\lambda_{1}^{+}\right)^{*}\right) R_{1}^{-1}(x, t)\left(n_{0,1}^{2}\left(n_{0,1}^{1}\right)^{*} \mathrm{e}^{\mathrm{i}\left(M_{1}^{-}(-x, t)\right)^{*}}-n_{0,1}^{5}\left(n_{0,1}^{4}\right)^{*} \mathrm{e}^{\mathrm{i} M_{1}^{+}(x, t)}\right), \\
& q_{31}^{-}(x, t)=-q_{53}^{-}(x, t)=-\left(\lambda_{1}^{+}+\left(\lambda_{1}^{+}\right)^{*}\right) R_{1}^{-1}(x, t)\left(n_{0,1}^{3}\left(n_{0,1}^{1}\right)^{*} \mathrm{e}^{\mathrm{i}\left(M_{1}^{-}(-x, t)\right)^{*}}+n_{0,1}^{5}\left(n_{0,1}^{3}\right)^{*} \mathrm{e}^{\mathrm{i} M_{1}^{+}(x, t)}\right), \\
& q_{41}^{-}(x, t)=q_{52}^{-}(x, t)=-\left(\lambda_{1}^{+}+\left(\lambda_{1}^{+}\right)^{*}\right) R_{1}^{-1}(x, t)\left(n_{0,1}^{4}\left(n_{0,1}^{1}\right)^{*} \mathrm{e}^{\mathrm{i}\left(M_{1}^{+}(-x, t)\right)^{*}}-n_{0,1}^{5}\left(n_{01}^{2}\right)^{*} \mathrm{e}^{\mathrm{i} M_{1}^{+}(x, t)}\right) .
\end{aligned}
$$

Here,

$$
\begin{aligned}
\left(M_{1}^{+}(-x, t)\right)^{*} & =\left(-\lambda_{1}^{+}\right)^{*}(-x)+2\left(\mathrm{f}\left(-\lambda_{1}^{+}\right)^{*}\right) \mathrm{t}, \quad \mathrm{R}_{1}(\mathrm{x}, \mathrm{t})=2 \mathrm{R}_{0,1} \cosh \left(2 \nu_{1}(\mathrm{x})+2 \tilde{\mu}_{1} \mathrm{t}+\xi_{0,1}\right)+\mathrm{S} \\
\nu_{1} & =\mathrm{i}\left(\left(-\lambda_{1}^{+}\right)^{*}-\lambda_{1}^{+}\right) / 2, \quad \tilde{\mu_{1}}=\mathrm{i}\left(\mathrm{f}\left(-\lambda_{1}^{+}\right)^{*}-\mathrm{f}\left(\lambda_{1}^{+}\right)\right) \\
S & =\left(n_{0,1}^{2}\right)^{*} n_{0,1}^{2}+\left(n_{0,1}^{3}\right)^{*} n_{0,1}^{3}+\left(n_{0,1}^{4}\right)^{*} n_{0,1}^{4} \\
R_{0,1} & =\sqrt{\left(n_{0,1}^{1}\right)^{*} n_{0,1}^{1}+\left(n_{0,1}^{5}\right)^{*} n_{0,1}^{5}}, \quad \xi_{0,1}=\frac{1}{2} \ln \frac{\left(\mathrm{n}_{0,1}^{1}\right)^{*} \mathrm{n}_{0,1}^{1}}{\left(\mathrm{n}_{0,1}^{5}\right)^{*} \mathrm{n}_{0,1}^{5}}
\end{aligned}
$$

Example 4. If we take again $\mathfrak{g} \simeq s o(5, \mathbb{C})$ and another $\mathbb{Z}_{2}$ reduction of type $(B)$ (16) with a block matrix $B$ containing an off-diagonal block $\mathbf{B}_{ \pm}$: $\mathbf{B}_{+}=\mathbf{B}_{-}=\mp 1$ and $\mathbf{B}_{ \pm}=\operatorname{off}$-diag $( \pm 1, \ldots, \pm 1)$. In this case, the reductions conditions for the matrix blocks can be written as:

$$
\mathbf{q}^{-}(x, t)=-\mathbf{B}_{-}\left(\mathbf{q}^{+}(-x, t)\right)^{\dagger}\left(\mathbf{B}_{+}\right)^{-1}, \quad \mathbf{q}^{+}(x, t)=-\mathbf{B}_{+}\left(\mathbf{q}^{-}(-x, t)\right)^{\dagger}\left(\mathbf{B}_{-}\right)^{-1} .
$$

Now, recall example A from Section 2, if we apply the involution of type $(B)$ with using $\mathbf{B}_{+}=\mathbf{B}_{-}=$ $\mp 1$ and $\mathbf{B}_{ \pm}=\operatorname{off}$-diag $( \pm 1, \ldots, \pm 1)$, then the 3-component $N L S$ system in (24) can be rewritten as:

$$
\begin{aligned}
& \mathrm{i}\left(q_{t}^{+}\right)_{12}+\left(q_{x x}^{+}\right)_{12}+2\left(q_{12}(x, t) q_{14}^{*}(-x, t)+2 q_{13}(x, t) q_{13}^{*}(-x, t)\right) q_{12}+2 q_{12}^{+, *}(-x, t)\left(q_{13}^{+}\right)^{2}=0, \\
& \mathrm{i}\left(q_{t}^{+}\right)_{13}+\left(q_{x x}^{+}\right)_{13}+2\left(q_{12}(x, t) q_{14}^{*}(-x, t)+q_{13}(x, t) q_{13}^{*}(-x, t)+q_{14}(x, t) q_{12}^{*}(-x, t)\right) q_{13} \\
& +2 q_{13}^{+, *}(-x, t) q_{14}^{+} q_{12}^{+}=0, \\
& \mathrm{i}\left(q_{t}^{+}\right)_{14}+\left(q_{x x}^{+}\right)_{14}+2\left(q_{14}(x, t) q_{12}^{*}(-x, t)+2 q_{13}(x, t) q_{13}^{*}(-x, t)\right) q_{14}+2 q_{14}^{+, *}(-x, t)\left(q_{13}^{+}\right)^{2}=0 .
\end{aligned}
$$


Also, we can write one-soliton solution of this system for this type of involution as the dressing factor (60) is satisfied by the involution (71) with (72). So, from (61), the components of the one-soliton solution can be written as:

$$
\begin{aligned}
& q_{12}^{+}(x, t)=q_{45}^{+}(x, t)=-\left(\lambda_{1}^{+}+\left(\lambda_{1}^{+}\right)^{*}\right) R_{1}^{-1}(x, t)\left(n_{0,1}^{1}\left(n_{0,1}^{4}\right)^{*} \mathrm{e}^{-\mathrm{i} M_{1}^{+}(x, t)}-n_{0,1}^{4}\left(n_{0,1}^{5}\right)^{*} \mathrm{e}^{-\mathrm{i}\left(M_{1}^{+}(-x, t)\right)^{*}}\right), \\
& q_{13}^{+}(x, t)=-q_{35}^{+}(x, t)=-\left(\lambda_{1}^{+}+\left(\lambda_{1}^{+}\right)^{*}\right) R_{1}^{-1}(x, t)\left(n_{0,1}^{1}\left(n_{0,1}^{3}\right)^{*} \mathrm{e}^{-\mathrm{i} M_{1}^{+}(x, t)}+n_{0,1}^{3}\left(n_{0,1}^{5}\right)^{*} \mathrm{e}^{-\mathrm{i}\left(M_{1}^{+}(-x, t)\right)^{*}}\right), \\
& q_{14}^{+}(x, t)=q_{25}^{+}(x, t)=-\left(\lambda_{1}^{+}+\left(\lambda_{1}^{+}\right)^{*}\right) R_{1}^{-1}(x, t)\left(n_{0,1}^{1}\left(n_{0,1}^{2}\right)^{*} \mathrm{e}^{-\mathrm{i} M_{1}^{+}(x, t)}-n_{0,1}^{2}\left(n_{0,1}^{5}\right)^{*} \mathrm{e}^{-\mathrm{i}\left(M_{1}^{+}(-x, t)\right)^{*}}\right), \\
& q_{21}^{-}(x, t)=q_{54}^{-}(x, t)=-\left(\lambda_{1}^{+}+\left(\lambda_{1}^{+}\right)^{*}\right) R_{1}^{-1}(x, t)\left(n_{0,1}^{2}\left(n_{0,1}^{1}\right)^{*} \mathrm{e}^{\mathrm{i}\left(M_{1}^{-}(-x, t)\right)^{*}}-n_{0,1}^{5}\left(n_{0,1}^{2}\right)^{*} \mathrm{e}^{\mathrm{i} M_{1}^{+}(x, t)}\right), \\
& q_{31}^{-}(x, t)=-q_{53}^{-}(x, t)=-\left(\lambda_{1}^{+}+\left(\lambda_{1}^{+}\right)^{*}\right) R_{1}^{-1}(x, t)\left(n_{0,1}^{3}\left(n_{0,1}^{1}\right)^{*} \mathrm{e}^{\mathrm{i}\left(M_{1}^{-}(-x, t)\right)^{*}}+n_{0,1}^{5}\left(n_{0,1}^{3}\right)^{*} \mathrm{e}^{\mathrm{i} M_{1}^{+}(x, t)}\right), \\
& q_{41}^{-}(x, t)=q_{52}^{-}(x, t)=-\left(\lambda_{1}^{+}+\left(\lambda_{1}^{+}\right)^{*}\right) R_{1}^{-1}(x, t)\left(n_{0,1}^{4}\left(n_{0,1}^{1}\right)^{*} \mathrm{e}^{\mathrm{i}\left(M_{1}^{+}(-x, t)\right)^{*}}-n_{0,1}^{5}\left(n_{01}^{4}\right)^{*} \mathrm{e}^{\mathrm{i} M_{1}^{+}(x, t)}\right) .
\end{aligned}
$$

Here,

$$
\begin{aligned}
\left(M_{1}^{+}(-x, t)\right)^{*} & =\left(-\lambda_{1}^{+}\right)^{*}(-x)+2\left(\mathrm{f}\left(-\lambda_{1}^{+}\right)^{*}\right) \mathrm{t}, \quad \mathrm{R}_{1}(\mathrm{x}, \mathrm{t})=2 \mathrm{R}_{0,1} \cosh \left(2 \nu_{1}(\mathrm{x})+2 \tilde{\mu}_{1} \mathrm{t}+\xi_{0,1}\right)+\mathrm{S} \\
\nu_{1} & =\mathrm{i}\left(\left(-\lambda_{1}^{+}\right)^{*}-\lambda_{1}^{+}\right) / 2, \quad \tilde{\mu_{1}}=\mathrm{i}\left(\mathrm{f}\left(-\lambda_{1}^{+}\right)^{*}-\mathrm{f}\left(\lambda_{1}^{+}\right)\right) \\
S & =\left(n_{0,1}^{4}\right)^{*} n_{0,1}^{2}+\left(n_{0,1}^{3}\right)^{*} n_{0,1}^{3}+\left(n_{0,1}^{2}\right)^{*} n_{0,1}^{4}, \\
R_{0,1} & =\sqrt{\left(n_{0,1}^{1}\right)^{*} n_{0,1}^{1}+\left(n_{0,1}^{5}\right)^{*} n_{0,1}^{5}}, \quad \xi_{0,1}=\frac{1}{2} \ln \frac{\left(\mathrm{n}_{0,1}^{1}\right)^{*} \mathrm{n}_{0,1}^{1}}{\left(\mathrm{n}_{0,1}^{5}\right)^{*} \mathrm{n}_{0,1}^{5}}
\end{aligned}
$$

\subsection{Two Soliton Solution}

Example 5. For $\mathfrak{g} \simeq \operatorname{sl}(3, \mathbb{C})$, in order to get the 2-soliton solution, the dressing factor $c$ (48) can be rewritten as:

$$
\begin{aligned}
\eta^{+}(x, t, \lambda) & =u_{1,2}(x, t, \lambda) \eta_{0}^{+}(x, t, \lambda)\left(\omega_{1,2}^{ \pm}(\lambda)\right)^{-1}, \\
u_{1,2}(x, t, \lambda) & =\mathbb{1}+\left(c_{1}(\lambda)-1\right) P_{1}(x, t)+\left(c_{2}(\lambda)-1\right) P_{2}(x, t) .
\end{aligned}
$$

Thus, the singular solution (two soliton solution) with singularities located at $\lambda_{1}^{ \pm}$and $\lambda_{2}^{ \pm}$can be obtained as follows:

$$
Q(x, t)=Q_{0}(x, t)-\sum_{k=1}^{2}\left(\lambda_{k}^{+}-\lambda_{k}^{-}\right)\left[\mathrm{J}, P_{k}(x, t)\right] .
$$

Assuming a canonical reduction of type (B) (16) with $\mathbf{B}=\mathbb{I}$, from (80) the components of the twosoliton solution can be written as:

$$
\begin{aligned}
& q_{1 j}(x, t)=-2 \sum_{k=1}^{2}\left(\lambda_{k}^{+}+\left(\lambda_{k}^{+}\right)^{*}\right) \frac{n_{0, k}^{k}\left(n_{0, k}^{j}\right)^{*} \mathrm{e}^{-\mathrm{i}\left(\tilde{M}_{k}^{+}(x, t)+\left(\tilde{M}_{k}^{+}\right)^{*}(-x, t)\right)}}{2 R_{0, k} \cosh \left(2 \nu_{\mathrm{k}} \mathrm{x}+2 \tilde{\left.\mu_{\mathrm{k}} \mathrm{t}+\xi_{0, \mathrm{k}}\right)}\right.}, \quad j=2,3, \\
& q_{j 1}(x, t)=2 \sum_{k=1}^{2}\left(\lambda_{k}^{+}+\left(\lambda_{k}^{+}\right)^{*}\right) \frac{n_{0, k}^{j}\left(n_{0, k}^{1}\right)^{*} \mathrm{e}^{\mathrm{i}\left(\tilde{M}_{k}^{+}(x, t)+\left(\tilde{M}_{k}^{+}\right)^{*}(-x, t)\right)}}{2 R_{0, k} \cosh \left(2 \nu_{\mathrm{k}} \mathrm{x}+2 \tilde{\mu_{\mathrm{k}}} \mathrm{t}+\xi_{0, \mathrm{k}}\right)}, \quad j=2,3 .
\end{aligned}
$$


Here,

$$
\begin{aligned}
\tilde{M}_{k}^{ \pm}(x, t) & =\lambda_{k}^{ \pm} x+2 \mathrm{f}_{0, \mathrm{k}}^{ \pm} \mathrm{t}, \quad\left(\tilde{\mathrm{M}}_{\mathrm{k}}^{+}(-\mathrm{x}, \mathrm{t})\right)^{*}=\left(-\lambda_{\mathrm{k}}^{+}\right)^{*}(-\mathrm{x})+2\left(\mathrm{f}\left(-\lambda_{1}^{+}\right)^{*}\right) \mathrm{t}, \\
\nu_{k} & =\mathrm{i}\left(\left(-\lambda_{\mathrm{k}}^{+}\right)^{*}-\lambda_{\mathrm{k}}^{+}\right) / 2, \quad \tilde{\mu_{\mathrm{k}}}=\mathrm{i}\left(\mathrm{f}\left(-\lambda_{\mathrm{k}}^{+}\right)^{*}-\mathrm{f}\left(\lambda_{\mathrm{k}}^{+}\right)\right), \\
R_{0, k} & =\sqrt{\left(n_{0, k}^{1}\right)^{*} n_{0, k}^{1}\left(\left(n_{0, k}^{2}\right)^{*} n_{0, k}^{2}+\left(n_{0, k}^{3}\right)^{*} n_{0, k}^{3}\right)}, \quad \xi_{0, k}=\frac{1}{2} \ln \frac{\left(\mathrm{n}_{0, \mathrm{k}}^{1}\right)^{*} \mathrm{n}_{0, \mathrm{k}}^{1}}{\left(\mathrm{n}_{0, \mathrm{k}}^{2}\right)^{*} \mathrm{n}_{0, \mathrm{k}}^{2}+\left(\mathrm{n}_{0, \mathrm{k}}^{3}\right)^{*} \mathrm{n}_{0, \mathrm{k}}^{3}} .
\end{aligned}
$$

Remark 1. Alternatively, in order to obtain 2-soliton solution, one can apply dressing method again on the 1-soliton solution used as a seed solution:

$$
\eta^{+}(x, t, \lambda)=u_{2}(x, t, \lambda) \eta_{1}^{+}(x, t, \lambda)\left(\omega_{2}^{ \pm}(\lambda)\right)^{-1}, \quad u_{2}(x, t, \lambda)=\mathbb{1}+\left(c_{2}(\lambda)-1\right) P_{2}(x, t) .
$$

Example 6. In the case of the involution of Example 2, the 2-soliton dressing factor (79a) is automatically compatible with the involution. Therefore, from (80), the components of the two-soliton solution can be written as:

$$
\begin{aligned}
& q_{12}(x, t)=-2 \sum_{k=1}^{2}\left(\lambda_{k}^{+}+\left(\lambda_{k}^{+}\right)^{*}\right) n_{0, k}^{1} \mathrm{e}^{-\mathrm{i} \tilde{M}_{k}^{+}(x, t)} R_{k}^{-1}(x, t)\left(n_{0, k}^{3}\right)^{*} \mathrm{e}^{-\mathrm{i} \tilde{M}_{k}^{+, *}(-x, t)}, \\
& q_{13}(x, t)=-2 \sum_{k=1}^{2}\left(\lambda_{k}^{+}+\left(\lambda_{k}^{+}\right)^{*}\right) n_{0, k}^{1} \mathrm{e}^{-\mathrm{i} \tilde{M}_{k}^{+}(x, t)} R_{k}^{-1}(x, t)\left(n_{0, k}^{2}\right)^{*} \mathrm{e}^{-\mathrm{i} \tilde{M}_{k}^{+, *}(-x, t)}, \\
& q_{21}(x, t)=-2 \sum_{k=1}^{2}\left(\lambda_{k}^{+}+\left(\lambda_{k}^{+}\right)^{*}\right) n_{0, k}^{2} \mathrm{e}^{\mathrm{i} \tilde{M}_{k}^{+}(x, t)} R_{k}^{-1}(x, t)\left(n_{0, k}^{1}\right)^{*} \mathrm{e}^{\left.\mathrm{i} \tilde{M}_{k}^{+, *}(-x, t)\right)}, \\
& q_{31}(x, t)=-2 \sum_{k=1}^{2}\left(\lambda_{k}^{+}+\left(\lambda_{k}^{+}\right)^{*}\right) n_{0, k}^{3} \mathrm{e}^{\mathrm{i} \tilde{M}_{k}^{+}(x, t)} R_{k}^{-1}(x, t)\left(n_{0, k}^{1}\right)^{*} \mathrm{e}^{\left.\mathrm{i} \tilde{M}_{k}^{+, *}(-x, t)\right)} .
\end{aligned}
$$

Here,

$$
\begin{aligned}
\tilde{M}_{k}^{ \pm}(x, t) & =\lambda_{k}^{ \pm} x+2 \mathrm{f}_{0, \mathrm{k}}^{ \pm} \mathrm{t}, \quad\left(\tilde{\mathrm{M}}_{\mathrm{k}}^{+}(-\mathrm{x}, \mathrm{t})\right)^{*}=\left(-\lambda_{\mathrm{k}}^{+}\right)^{*}(-\mathrm{x})+2\left(\mathrm{f}\left(-\lambda_{1}^{+}\right)^{*}\right) \mathrm{t} \\
R_{1}(x, t) & =2 R_{0,1} \cosh \left(2 \nu_{1} \mathrm{x}+2 \tilde{\mu}_{1} \mathrm{t}+\xi_{0,1}\right), \\
\nu_{k} & =\mathrm{i}\left(\left(-\lambda_{\mathrm{k}}^{+}\right)^{*}-\lambda_{\mathrm{k}}^{+}\right) / 2, \quad \tilde{\mu_{\mathrm{k}}}=\mathrm{i}\left(\mathrm{f}\left(-\lambda_{\mathrm{k}}^{+}\right)^{*}-\mathrm{f}\left(\lambda_{\mathrm{k}}^{+}\right)\right) \\
R_{0, k} & =\sqrt{\left(n_{0, k}^{1}\right)^{*} n_{0, k}^{1}\left(\left(n_{0, k}^{3}\right)^{*} n_{0, k}^{2}+\left(n_{0, k}^{2}\right)^{*} n_{0, k}^{3}\right)}, \quad \xi_{0, k}=\frac{1}{2} \ln \frac{\left(\mathrm{n}_{0, \mathrm{k}}^{1}\right)^{*} \mathrm{n}_{0, \mathrm{k}}^{1}}{\left(\mathrm{n}_{0, \mathrm{k}}^{3}\right)^{*} \mathrm{n}_{0, \mathrm{k}}^{2}+\left(\mathrm{n}_{0, \mathrm{k}}^{2}\right)^{*} \mathrm{n}_{0, \mathrm{k}}^{3}} .
\end{aligned}
$$

Example 7. If we take $\mathfrak{g} \simeq s o(5, \mathbb{C})$, then the 2 -soliton dressing factors $u(x, t, \lambda)$ can be considered with two more poles in the following form:

$$
\begin{gathered}
u_{1,2}(x, t, \lambda)=\mathbb{1}+\left(c_{1}(\lambda)-1\right) P_{1}(x, t)+\left(\frac{1}{c_{1}(\lambda)}-1\right) \bar{P}_{1}(x, t), \\
+\left(c_{2}(\lambda)-1\right) P_{2}(x, t)+\left(\frac{1}{c_{2}(\lambda)}-1\right) \bar{P}_{2}(x, t), \\
c_{k}(\lambda)=\frac{\lambda-\lambda_{k}^{+}}{\lambda-\lambda_{k}^{-}}, \quad P_{k}(x, t)=\frac{\left|n_{k}(x, t)\right\rangle\left\langle m_{k}(x, t)\right|}{\left\langle m_{k}(x, t) \mid n_{k}(x, t)\right\rangle}, \quad \bar{P}_{k}=S_{0} P_{k}^{T} S_{0}^{-1}, \quad k=1,2 .
\end{gathered}
$$


Thus, the new potential $Q(x, t)$ corresponding to a given trivial solution $Q_{0}(x, t)=0$ can be obtained by the following form:

$$
Q(x, t)=-\sum_{k=1}^{2}\left(\lambda_{k}^{+}-\lambda_{k}^{-}\right)\left[\mathrm{J}, P_{k}(x, t)-\bar{P}_{k}(x, t)\right]
$$

The components of new potential $Q(x, t)$ can be obtained by:

$$
\begin{gathered}
q_{12}^{+}(x, t)=q_{45}^{+}(x, t)=-\sum_{k=1}^{2}\left(\lambda_{k}^{+}-\lambda_{k}^{-}\right) R_{k}^{-1}(x, t)\left(n_{0, k}^{1} m_{0, k}^{2} \mathrm{e}^{-\mathrm{i} M_{k}^{+}(x, t)}+n_{0, k}^{4} m_{0, k}^{5} \mathrm{e}^{-\mathrm{i} M_{k}^{-}(x, t)}\right), \\
q_{13}^{+}(x, t)=-q_{35}^{+}(x, t)=-\sum_{k=1}^{2}\left(\lambda_{k}^{+}-\lambda_{k}^{-}\right) R_{k}^{-1}(x, t)\left(n_{0, k}^{1} m_{0, k}^{3} \mathrm{e}^{-\mathrm{i} M_{k}^{+}(x, t)}-n_{0, k}^{3} m_{0, k}^{5} \mathrm{e}^{-\mathrm{i} M_{k}^{-}(x, t)}\right), \\
q_{14}^{+}(x, t)=q_{25}^{+}(x, t)=-\sum_{k=1}^{2}\left(\lambda_{k}^{+}-\lambda_{k}^{-}\right) R_{k}^{-1}(x, t)\left(n_{0, k}^{1} m_{0, k}^{4} \mathrm{e}^{-\mathrm{i} M_{k}^{+}(x, t)}+n_{0, k}^{2} m_{0, k}^{5} \mathrm{e}^{-\mathrm{i} M_{k}^{-}(x, t)}\right), \\
q_{21}^{-}(x, t)=q_{54}^{-}(x, t)=-\sum_{k=1}^{2}\left(\lambda_{k}^{+}-\lambda_{k}^{-}\right) R_{k}^{-1}(x, t)\left(-n_{0, k}^{2} m_{0, k}^{1} \mathrm{e}^{\mathrm{i} M_{k}^{-}(x, t)}-n_{0, k}^{5} m_{0, k}^{4} \mathrm{e}^{\mathrm{i} M_{k}^{+}(x, t)}\right), \\
q_{31}^{-}(x, t)=-q_{53}^{-}(x, t)=-\sum_{k=1}^{2}\left(\lambda_{k}^{+}-\lambda_{k}^{-}\right) R_{k}^{-1}(x, t)\left(-n_{0, k}^{3} m_{0, k}^{1} \mathrm{e}^{\mathrm{i} M_{k}^{-}(x, t)}+n_{0, k}^{5} m_{0, k}^{3} \mathrm{e}^{\mathrm{i} M_{k}^{+}(x, t)}\right), \\
q_{41}^{-}(x, t)=q_{52}^{-}(x, t)=-\sum_{k=1}^{2}\left(\lambda_{k}^{+}-\lambda_{k}^{-}\right) R_{k}^{-1}(x, t)\left(-n_{0, k}^{4} m_{0, k}^{1} \mathrm{e}^{\mathrm{i} M_{k}^{-}(x, t)}-n_{0, k}^{5} m_{0, k}^{2} \mathrm{e}^{\mathrm{i} M_{k}^{+}(x, t)}\right) .
\end{gathered}
$$

Here

$$
\begin{aligned}
M_{k}^{ \pm}(x, t) & =\lambda_{k}^{ \pm} x+2 f_{0, k}^{ \pm} t, \quad R_{k}(x, t)=2 R_{0, k} \cosh \left(2 \nu_{\mathrm{k}} \mathrm{x}+2 \tilde{\mu}_{\mathrm{k}} \mathrm{t}+\xi_{0, \mathrm{k}}\right)+\mathrm{S} \\
\nu_{k} & =\mathrm{i}\left(\lambda_{\mathrm{k}}^{-}-\lambda_{\mathrm{k}}^{+}\right) / 2, \quad \tilde{\mu_{\mathrm{k}}}=\mathrm{i}\left(\mathrm{f}\left(\lambda_{\mathrm{k}}^{-}\right)-\mathrm{f}\left(\lambda_{\mathrm{k}}^{+}\right)\right), \quad \mathrm{S}=\mathrm{m}_{0, \mathrm{k}}^{2} \mathrm{n}_{0, \mathrm{k}}^{2}+\mathrm{m}_{0, \mathrm{k}}^{3} \mathrm{n}_{0, \mathrm{k}}^{3}+\mathrm{m}_{0, \mathrm{k}}^{4} \mathrm{n}_{0, \mathrm{k}}^{4}, \\
R_{0, k} & =\sqrt{m_{0, k}^{1} n_{0, k}^{1}+m_{0, k}^{5} n_{0, k}^{5}}, \quad \xi_{0, k}=\frac{1}{2} \ln \frac{\mathrm{m}_{0, \mathrm{k}}^{1} \mathrm{n}_{0, \mathrm{k}}^{1}}{\mathrm{~m}_{0, \mathrm{k}}^{5} \mathrm{n}_{0, \mathrm{k}}^{5}}
\end{aligned}
$$

Example 8. Again in the case when $\mathfrak{g} \simeq s o(5, \mathbb{C})$, if we impose a nonlocal involution of the form $(16)$ then the dressing factor in (84) will satisfy the following reduction condition:

$$
B \prod_{k=1}^{2} u_{k}\left(-x, t,-\lambda^{*}\right)^{\dagger} B^{-1}=\prod_{k=1}^{2} u_{k}^{-1}(x, t, \lambda)
$$

where B is constant block-diagonal matrix. This involution is satisfied if:

$$
P_{k}(x, t)=B P_{k}^{\dagger}(-x, t) B^{-1}, \quad\left(-\lambda^{ \pm}\right)^{*}=\lambda^{\mp} .
$$

That means the projector $P_{k}(x, t)$ and $c_{k}(\lambda)$ become:

$$
P_{k}(x, t)=\frac{\left|n_{k}(x, t)\right\rangle\left\langle n_{k}^{*}(-x, t)\right| B}{\left\langle n_{k}^{*}(-x, t)|B| n_{k}(x, t)\right\rangle}, \quad\left\langle m_{k}(x, t)\right|=\left(B\left|n_{k}(-x, t)\right\rangle\right)^{\dagger}, \quad c_{k}(\lambda)=\frac{\lambda-\lambda_{k}^{+}}{\lambda+\left(\lambda_{k}^{+}\right)^{*}} .
$$

So, from (61), it follows that

$$
Q(x, t)=Q_{0}(x, t)-\left(\lambda_{k}^{+}+\left(\lambda_{k}^{+}\right)^{*}\right)\left[\mathrm{J}, B P_{k}^{\dagger}(-x, t) B^{-1}\right] .
$$


Thus, the components of two soliton solution can be written as:

$$
\begin{aligned}
& q_{12}^{+}(x, t)=q_{45}^{+}(x, t)=-\sum_{k=1}^{2}\left(\lambda_{k}^{+}+\left(\lambda_{k}^{+}\right)^{*}\right) R_{k}^{-1}(x, t)\left(n_{0, k}^{1}\left(n_{0, k}^{2}\right)^{*} \mathrm{e}^{-\mathrm{i} M_{k}^{+}(x, t)}-n_{0, k}^{4}\left(n_{0, k}^{5}\right)^{*} \mathrm{e}^{-\mathrm{i}\left(M_{k}^{+}(-x, t)\right)^{*}}\right), \\
& q_{13}^{+}(x, t)=-q_{35}^{+}(x, t)=-\sum_{k=1}^{2}\left(\lambda_{k}^{+}+\left(\lambda_{k}^{+}\right)^{*}\right) R_{k}^{-1}(x, t)\left(n_{0, k}^{1}\left(n_{0, k}^{3}\right)^{*} \mathrm{e}^{-\mathrm{i} M_{k}^{+}(x, t)}+n_{0, k}^{3}\left(n_{0, k}^{5}\right)^{*} \mathrm{e}^{-\mathrm{i}\left(M_{k}^{+}(-x, t)\right)^{*}}\right), \\
& q_{14}^{+}(x, t)=q_{25}^{+}(x, t)=-\sum_{k=1}^{2}\left(\lambda_{k}^{+}+\left(\lambda_{k}^{+}\right)^{*}\right) R_{k}^{-1}(x, t)\left(n_{0, k}^{1}\left(n_{0, k}^{4}\right)^{*} \mathrm{e}^{-\mathrm{i} M_{k}^{+}(x, t)}-n_{0, k}^{2}\left(n_{0, k}^{5}\right)^{*} \mathrm{e}^{-\mathrm{i}\left(M_{k}^{+}(-x, t)\right)^{*}}\right), \\
& q_{21}^{-}(x, t)=q_{54}^{-}(x, t)=-\sum_{k=1}^{2}\left(\lambda_{k}^{+}+\left(\lambda_{k}^{+}\right)^{*}\right) R_{k}^{-1}(x, t)\left(n_{0, k}^{2}\left(n_{0, k}^{1}\right)^{*} \mathrm{e}^{\mathrm{i}\left(M_{k}^{-}(-x, t)\right)^{*}}-n_{0, k}^{5}\left(n_{0, k}^{4}\right)^{*} \mathrm{e}^{\mathrm{i} M_{k}^{+}(x, t)}\right), \\
& q_{31}^{-}(x, t)=-q_{53}^{-}(x, t)=-\sum_{k=1}^{2}\left(\lambda_{k}^{+}+\left(\lambda_{k}^{+}\right)^{*}\right) R_{k}^{-1}(x, t)\left(n_{0, k}^{3}\left(n_{0, k}^{1}\right)^{*} \mathrm{e}^{\mathrm{i}\left(M_{k}^{-}(-x, t)\right)^{*}}+n_{0, k}^{5}\left(n_{0, k}^{3}\right)^{*} \mathrm{e}^{\mathrm{i} M_{k}^{+}(x, t)}\right), \\
& q_{41}^{-}(x, t)=q_{52}^{-}(x, t)=-\sum_{k=1}^{2}\left(\lambda_{k}^{+}+\left(\lambda_{k}^{+}\right)^{*}\right) R_{k}^{-1}(x, t)\left(n_{0, k}^{4}\left(n_{0, k}^{1}\right)^{*} \mathrm{e}^{\mathrm{i}\left(M_{k}^{+}(-x, t)\right)^{*}}-n_{0, k}^{5}\left(n_{0, k}^{2}\right)^{*} \mathrm{e}^{\mathrm{i} M_{k}^{+}(x, t)}\right) .
\end{aligned}
$$

Here,

$$
\begin{aligned}
\left(M_{k}^{+}(-x, t)\right)^{*} & =\left(-\lambda_{k}^{+}\right)^{*}(-x)+2\left(\mathrm{f}\left(-\lambda_{1}^{+}\right)^{*}\right) \mathrm{t}, \quad \mathrm{R}_{\mathrm{k}}(\mathrm{x}, \mathrm{t})=2 \mathrm{R}_{0, \mathrm{k}} \cosh \left(2 \nu_{\mathrm{k}}(\mathrm{x})+2 \tilde{\mu_{\mathrm{k}}} \mathrm{t}+\xi_{0, \mathrm{k}}\right)+\mathrm{S} \\
\nu_{k} & =\mathrm{i}\left(\left(-\lambda_{\mathrm{k}}^{+}\right)^{*}-\lambda_{\mathrm{k}}^{+}\right) / 2, \quad \tilde{\mu_{\mathrm{k}}}=\mathrm{i}\left(\mathrm{f}\left(-\lambda_{\mathrm{k}}^{+}\right)^{*}-\mathrm{f}\left(\lambda_{\mathrm{k}}^{+}\right)\right), \\
S & =\left(n_{0, k}^{2}\right)^{*} n_{0, k}^{2}+\left(n_{0, k}^{3}\right)^{*} n_{0, k}^{3}+\left(n_{0, k}^{4}\right)^{*} n_{0, k}^{4}, \\
R_{0, k} & =\sqrt{\left(n_{0, k}^{1}\right)^{*} n_{0, k}^{1}+\left(n_{0, k}^{5}\right)^{*} n_{0, k}^{5}}, \quad \xi_{0, k}=\frac{1}{2} \ln \frac{\left(\mathrm{n}_{0, \mathrm{k}}^{1}\right)^{*} \mathrm{n}_{0, \mathrm{k}}^{1}}{\left(\mathrm{n}_{0, \mathrm{k}}^{5}\right)^{*} \mathrm{n}_{0, \mathrm{k}}^{5}}
\end{aligned}
$$

\section{Conclusions}

We have studied here multi-component generalisations of NLS models on A.III and BD.I symmetric spaces. Our study is based on two types of main examples: the Manakov vector NLS equation, related to symmetric spaces of A.III type and Kulish-Sklyanin models, related to symmetric spaces of BD.I type. Firstly, we formulated the direct scattering problem for both models. This includes: the construction of the Jost solutions, the scattering matrix and the minimal set of scattering data. Based on the Gauss decomposition of the scattering matrix, we have also constructed the fundamental analytic solutions (FAS).

It turns out that the spectral properties of the Lax operator depend crucially on the choice of representation of the underlying Lie algebra or symmetric space while the minimal set of scattering data is provided by the same set of functions [28].

Finally, we have presented a modification of the dressing method and obtained 1- and 2-solitons for both models with nonlocal reductions. Depending on the positions of the discrete eigenvalues $\lambda_{j}^{ \pm}$in the spectral plane, there are two regimes for the 2-soliton solution: if two of the discrete eigenvalues are in the upper half of the complex plane, while the other two are in the lower half, then the nonlocal involution will preserve their number inside each of the contours, so it will result 
in a Riemann-Hilbert problem with balanced number of singularities and therefore the corresponding 2 -soliton solutions will be regular for all $t$. Otherwise, the 2-soliton solution will develop again a singularity in finite time as in the case, studied in $[2,3,4]$.

The results of this paper can be extended in several directions:

- To construct gauge covariant formulation of the multi-component NLS hierarchies on symmetric spaces, including the Wronskian relations, the "squared solutions" and their completeness relations, the descriptions of the class of NLEEs associated to a given scattering problem, the generating (recursion) operator and it spectral decomposition, the description of the infinite set of integrals of motion, the hierarchy of Hamiltonian structures and the $r$-matrix formulation.

- To study the gauge equivalent systems of multi-component ferromagnetics on symmetric spaces $[31,34,35]$.

- To study different types of reductions of multi-component integrable systems on symmetric spaces [44, 46, 47].

- To study the associated Darboux transformations and their generalizations for NLS equations over Hermitian symmetric spaces and to obtain multi-soliton solutions via such generalizations. This includes also rational solutions [13].

- To extend our results for the case of non-vanishing boundary conditions (a non-trivial background) $[5,51]$. The considerations required in this case are more complicated and will be discussed it elsewhere.

- To study other types of integrable hierarchies on symmetric spaces, e.g. quadratic bundles (related to the derivative NLS equation, the Kaup-Newell equation or Gerdjikov-Ivanov equation) $[32,33,41,58,59,38]$ or rational bundles [34, 35].

\section{Acknowledgements}

The authors have the pleasure to thank Prof. Mark Ablowitz and Prof. Vladimir Gerdjikov for numerous useful discussions.

\section{References}

[1] F.K. Abdullaev, Y.V. Kartashov, V.V. Konotop, D.A. Zezyulin, Solitons in PT-symmetric nonlinear lattices, Phys. Rev. A 83 (2011), 041805.

[2] M. Ablowitz and Z. Musslimani, Integrable Nonlocal Nonlinear Schrödinger Equation, Phys. Rev. Lett., 110 (2013) 064105.

[3] M. Ablowitz and Z. Musslimani, Integrable discrete PT symmetric model, Phys. Rev. E 90 (2014) 032912.

[4] MJ Ablowitz, ZH Musslimani, Inverse scattering transform for the integrable nonlocal nonlinear Schrödinger equation, Nonlinearity 29 (2016) 915-946.

[5] MJ Ablowitz, H-D Luo, ZH Musslimani, Inverse scattering transform for the nonlocal nonlinear Schrödinger equation with nonzero boundary conditions, E-print: arXiv: 1612.02726. 
[6] M. J. Ablowitz, B. Prinari, and A. D. Trubatch, Discrete and continuous nonlinear Schrödinger systems, (2005).

[7] J. R. Ackerhalt, P. W. Milonni, Solitons and four-wave mixing, Phys. Rev. A 33 (1986) 31853198.

[8] Athorne C., Fordy A., Generalised KdV and MKdV equations associated with symmetric spaces, J. Phys. A: Math. Gen. 20 (1987), 1377-1386.

[9] I. V. Barashenkov, Hamiltonian formulation of the standard $\mathcal{P} \mathcal{T}$-symmetric nonlinear Schrödinger dimer, Physical Review A 90 (2014) 045802.

[10] I V Barashenkov, D E Pelinovsky and P Dubard, Dimer with gain and loss: Integrability and $\mathcal{P} \mathcal{T}$-symmetry restoration, J. Phys. A: Math. Theor. 48 (2015) 325201.

[11] C. M. Bender and S. Boettcher, Real Spectra in Non-hermitian Hamiltonians Having PT Symmetry, Phys. Rev. Lett 80 (1998) 5243-5246;

C. M. Bender, S. Boettcher and P. N. Meisinger, PT-Symmetric quantum Mechanics, J. Math. Phys. 40 (1999) 2201-2229.

[12] C. M. Bender, Making Sense of Non-hermitian Hamiltonians, Rep. Progr. Phys. 70 (2007) 9471018 (E-print: hep-th/0703096).

[13] E. V. Doktorov, and S. B. Leble, Dressing method in Mathematical Physics, Mathematical Physics Studies 28, Springer, Berlin, Heidelberg, New York (2007).

[14] Beals R., Coifman R R., Scattering and inverse scattering for first order systems, Commun. Pure and Appl. Math. 37 (1984), n 1, 39-90;

Scattering and inverse scattering for first order systems II, Inverse Problems 3 (1987), 577-594.

[15] P. G. Drazin, , and R. S. Johnson, Solitons: an introduction. Vol. 2. Cambridge university press, 1989.

[16] V.V. Drinfel'd and V. V. Sokolov, Lie algebras and equations of Korteweg-de Vries type, Journal of Soviet Mathematics, 30 (1985) 1975-2036.

[17] L. D. Faddeev, L. A. Takhtadjan, Hamiltonian Method in the Theory of Solitons, Springer Verlag, Berlin (1987).

[18] Fordy A.P., Derivative nonlinear Schrödinger equations and Hermitian symmetric spaces, J. Phys. A: Math. Gen. 17 (1984), 1235-1245.

[19] Fordy A.P., Kulish P.P., Nonlinear Schrödinger equations and simple Lie algebras, Comm. Math. Phys. 89 (1983), 427-443.

[20] A Fring, $\mathcal{P} \mathcal{T}$-symmetric deformations of the Korteweg-de Vries equation, J. Phys. A: Math. Theor. 40 (2007), 4215 (E-print: math-ph / 0701036$).$

[21] A Fring, $\mathcal{P} \mathcal{T}$-symmetric deformations of integrable models, Phil. Trans. Royal Soc. A 371 (2013), 20120046.

[22] V. S. Gerdjikov, Generalised Fourier transforms for the soliton equations. Gauge covariant formulation, Inverse Problems 2 (1986), 51-74. 
[23] V. S. Gerdjikov, Basic aspects of soliton theory, Proceedings of the Sixth International Conference on Geometry, Integrability and Quantization. Institute of Biophysics and Biomedical Engineering, Bulgarian Academy of Sciences, 2005.

[24] V. S. Gerdjikov, Kulish-Sklyanin type models: integrability and reductions, arXiv preprint arXiv:1702.04010 (2017).

[25] V. S. Gerdjikov, On nonlocal models of Kulish-Sklyanin type and generalized Fourier transforms, Advanced Computing in Industrial Mathematics. Springer International Publishing, 2017. $37-52$.

[26] V. S. Gerdjikov, On Reductions of Soliton Solutions of Multi-component NLS Models and Spinor Bose-Einstein Condensates, AIP Conference Proceedings. Vol. 1186. No. 1. AIP, 2009.

[27] V. S. Gerdjikov, Algebraic and Analytic Aspects of $N$-wave Type Equations, Contemporary Mathematics 301 (2002) 35-68 (E-print: nlin. S I / 0206014 ).

[28] V. S. Gerdjikov and G. G. Grahovski, Multi-component NLS models on symmetric spaces: Spectral properties versus representations theory, SIGMA 6 (2010), paper 044 (29 pages).

[29] V. S. Gerdjikov, G. G. Grahovski, N. A. Kostov, Reductions of N-wave interactions related to low-rank simple Lie algebras. I: $\mathbb{Z}_{2}$ - reductions, J. Phys. A: Math \& Gen. 34 (2001) 9425-9461 (E-print: nlin.SI/0006001).

[30] V. S. Gerdjikov, G. G. Grahovski, R. I. Ivanov, N. A. Kostov, N-wave interactions related to simple Lie algebras. $\mathbb{Z}_{2}$ - reductions and soliton solutions, Inverse Problems 17 (2001) 999-1015 (E-print: nlin.SI/0009034).

[31] V. S. Gerdjikov, G. G. Grahovski, N. A. Kostov, On N-wave Type Systems and Their Gauge Equivalent, European J. Phys. B 29 (2002) 243-248.

[32] V. S. Gerdjikov, G. G. Grahovski and R. I. Ivanov, On the N-wave Equations with PT-symmetry, Theor. Math. Phys. 188 (2016), 1305-1321.

[33] V. S. Gerdjikov, G. G. Grahovski and R. I. Ivanov, On integrable wave interactions and Lax pairs on symmetric spaces, Wave Motion 71 (2017), 53-70.

[34] V. S. Gerdjikov, G. G. Grahovski, A. V. Mikhailov, T. I. Valchev, Rational bundles and recursion operators for integrable equations on A. III-type symmetric spaces, Theor. Math. Phys. 167 (2011), 740-750.

[35] V. S. Gerdjikov, G. G. Grahovski, A. V. Mikhailov, T. I. Valchev, Polynomial bundles and generalised Fourier transforms for integrable equations on A. III-type symmetric spaces, SIGMA 7 (2011), paper 096 (48 pages).

[36] V. S. Gerdjikov, D. J. Kaup, N. A. Kostov, and T. I. Valchev, On classification of soliton solutions of multicomponent nonlinear evolution equations, J. Phys. A: Math. Theor. 41 (2008) 315213 (36pp).

[37] VA Atanasov, VS Gerdjikov, GG Grahovski, NA Kostov, Fordy-Kulish model and spinor BoseEinstein condensate, J. Nonl. Math. Phys. 15 (2008), 291-298. 
[38] V.S. Gerdjikov, N.A. Kostov, T.I. Valchev, N-wave equations with orthogonal algebras: $\mathbb{Z}_{2}$ and $\mathbb{Z}_{2} \times \mathbb{Z}_{2}$ reductions and soliton solutions, SIGMA 3 (2007), paper 039 (19 pages) (E-print: nlin.SI/0703002).

[39] V. S. Gerdjikov, P. P. Kulish, The generating operator for the $n \times n$ linear system, Physica D 3 (1981) 549-564.

[40] V. S. Gerdjikov, A. Saxena, Complete Integrability of Nonlocal Nonlinear Schrödinger equation, J. Math. Phys. 58 (2017), 013502 (E-print: arXiv: 1510.0480 ).

[41] V. S. Gerdjikov, G. Vilasi, and A. B. Yanovski, Integrable Hamiltonian Hierarchies. Spectral and Geometric Methods, Lecture Notes in Physics 748, Springer Verlag, Berlin, Heidelberg, New York (2008).

[42] R. Gilmore, Lie groups, Lie algebras and some of their applications, Dover Publications, New York (2005).

[43] G. G. Grahovski, On the Reductions and Scattering Data for the Generalized Zakharov-Shabat Systems, In: "Nonlinear Physics: Theory and Experiment. II", Eds: M. J. Ablowitz, M. Boiti, F. Pempinelli and B. Prinari, World Scientific, Singapore (2003), pp. 71-78;

[44] G. G. Grahovski, Condon M., On the Caudrey-Beals-Coifman System and the Gauge Group Action, J. Nonlin. Math. Phys. 15 (2008), suppl. 3, 197-208 (E-print: arXiv: 0710.3302 ).

[45] G. G. Grahovski, V. S. Gerdjikov and Nikolay A, Kostov, On the multi-component NLS type equations on symmetric spaces: reductions and soliton solutions, In: Proc. Sixth International Conference on Geometry, Integrability and Quantization, Varna, Bulgaria Ivailo M. M ladenov and Allen C. Hirshfel, Editors, SOFTEX, Sofia (2005), pp. 203-217.

[46] G. G. Grahovski, V. S. Gerdjikov, N. A. Kostov and V. A. Atanasov, New Integrable MultiComponent NLS Type Equations on Symmetric Spaces: $\mathbb{Z}_{4}$ and $\mathbb{Z}_{6}$ Reductions, In: Proc. Seventh International Conference on Geometry, Integrability and Quantization, Varna, Bulgaria Ivailo M. M ladenov and Manuel de Leon, Editors, SOFTEX, Sofia (2006), pp. 154-175.

[47] M. Gürses, Nonlocal Fordy-Kulish equations on symmetric spaces, Phys. Lett. A 381 (2017), 1791-1794.

[48] Helgason S., Differential geometry, Lie groups and Symmetric Spaces, Graduate Studies in Mathematics 34, AMS, Providence, Rhode Island (2001).

[49] R. Ivanov, On the dressing method for the generalised Zakharov-Shabat system, Nuclear Phys. B 694 (2004) 509-524 (E-print: math-ph / 0402031 ).

[50] P. P. Kulish, and E. K. Sklyanin, $O(N)$-invariant nonlinear Schrödinger equation a new completely integrable system, Physics Letters A 84 (1981): 349-352.

[51] Min Li and Tao Xu, Dark and antidark soliton interactions in the nonlocal nonlinear Schrödinger equation with the self-induced parity-time-symmetric potential, Phys. Rev. E 91 (2015), 033202.

[52] Loos O., Symmetric spaces, vol. I: General theory; vol. II: Compact spaces and classification, W. A. Benjamin, Inc., New York-Amsterdam, 1969. 
[53] S. V. Manakov, On the theory of two-dimensional stationary self-focusing of electromagnetic waves, Sov. Phys. JETP 38 (1974) 248-255.

[54] C. R. Menyuk, Nonlinear pulse propagation in birefringent optical fibres, IEEE J. Quantum Electron. 23 (1987) 174-176.

[55] A. Mostafazadeh, Pseudo-hermiticity versus PT-Symmetry I, II, III, J. Math. Phys. 43 (2002) 205-214 (E-print: math-ph/0107001); 2814-2816 (E-print: math-ph/0110016); 39443951 (E-print: math-ph/0203005).

[56] A. Mostafazadeh, Pseudo-hermiticity and Generalized $\mathcal{P} \mathcal{T}$ - and $\mathcal{C} \mathcal{P} \mathcal{T}$-Symmetries, J. Math. Phys. 44 (2003) 974-989 (E-print: math-ph / 0209018 );

A. Mostafazadeh, Exact PT-Symmetry Is Equivalent to Hermiticity, J. Phys. A: Math. Gen. 36 (2003) 7081-7091 (E-print: quant-ph/0304080).

[57] A. V. Mikhailov, The reduction problem and the inverse scattering problem, Physica D 3 (1981) $73-117$.

[58] A. G. Reiman. A unified Hamiltonian system on polynomial bundles, and the structure of stationary problems. Sci. Notes of LOMI seminars vol. 131, pp. 118-127, (1983).

[59] A.G. Reiman, and M. A. Semenov-Tyan-Shanskii. Current algebras and nonlinear partial differential equations, Dokl. Akad. Nauk SSSR, 251, 1310-1312 (1980).

[60] C. E. Rüter, K. G. Makris, R. El-Ganainy, D. N. Christodoulides, M. Segev and D. Kip, Observation of parity-time symmetry in optics, Nature Physics 6 (2010) 192 - 195.

[61] Shabat A. B., Inverse scattering problem for a system of differential equations, Functional Annal. \& Appl. 9 (1975), 75-78 (In Russian);

Shabat A. B. The inverse scattering problem, Diff. Equations 15 (1979), 1824-1834.

[62] T. I. Valchev, On Mikhailov's reduction group, Phys. Lett A 379 (2015) 1877-1880.

[63] S. P. Novikov, S. V. Manakov, L. P. Pitaevskii, and V. E. Zakharov, Theory of solitons: the inverse scattering method, New York: Plenum Press (1984).

[64] Zakharov V. E. and Mikhailov A. V., On the integrability of classical spinor models in twodimensional space-time, Commun. Math. Phys. 74 (1980), 21-40.

[65] V. E. Zakharov, and A. B. Shabat, A scheme for integrating nonlinear evolution equations of mathematical physics by the inverse scattering method. I \& II, Funkts. Anal. Prilozhen., 8 (1974), 43-53; 13 (1979) no. 3, 13-22.

[66] A. A. Zyablovsky, A. P. Vinogradov, A. A. Pukhov, A. V. Dorofeenko and A A Lisyansky, $\mathcal{P} \mathcal{T}$-symmetry in optics, Phys.-Uspekhi 57 (2014), no. 11, 1063-1082. 